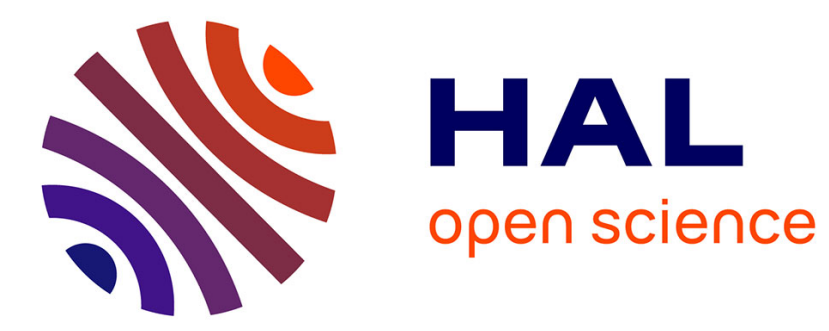

\title{
Probabilistic Algorithm for Computing the Dimension of Real Algebraic Sets
}

\author{
Ivan Bannwarth, Mohab Safey El Din
}

\section{To cite this version:}

Ivan Bannwarth, Mohab Safey El Din. Probabilistic Algorithm for Computing the Dimension of Real Algebraic Sets. ISSAC'15 - 40th International Symposium on Symbolic and Algebraic Computation, Jul 2015, Bath, United Kingdom. pp.37-44, 10.1145/2755996.2756670 . hal-01152751

\section{HAL Id: hal-01152751 \\ https://hal.science/hal-01152751}

Submitted on 19 May 2015

HAL is a multi-disciplinary open access archive for the deposit and dissemination of scientific research documents, whether they are published or not. The documents may come from teaching and research institutions in France or abroad, or from public or private research centers.
L'archive ouverte pluridisciplinaire HAL, est destinée au dépôt et à la diffusion de documents scientifiques de niveau recherche, publiés ou non, émanant des établissements d'enseignement et de recherche français ou étrangers, des laboratoires publics ou privés. 


\title{
Probabilistic Algorithm for Computing the Dimension of Real Algebraic Sets
}

\author{
Ivan Bannwarth, Mohab Safey El Din \\ Sorbonne Universités, Univ. Pierre et Marie \\ Curie (Paris 06) \\ INRIA Paris Rocquencourt, POLSYS Project \\ LIP6 CNRS, UMR 7606 \\ Ivan.Bannwarth@lip6.fr, Mohab.Safey@lip6.fr
}

\begin{abstract}
Let $f \in \mathbb{Q}\left[X_{1}, \ldots, X_{n}\right]$ be a polynomial of degree $D$. We consider the problem of computing the real dimension of the real algebraic set defined by $f=0$. Such a problem can be reduced to quantifier elimination. Hence it can be tackled with Cylindrical Algebraic Decomposition within a complexity that is doubly exponential in the number of variables. More recently, denoting by $d$ the dimension of the real algebraic set under study, deterministic algorithms running in time $D^{O(d(n-d))}$ have been proposed. However, no implementation reflecting this complexity gain has been obtained and the constant in the exponent remains unspecified.

We design a probabilistic algorithm which runs in time which is essentially cubic in $D^{d(n-d)}$. Our algorithm takes advantage of genericity properties of polar varieties to avoid computationally difficult steps of quantifier elimination. We also report on a first implementation. It tackles examples that are out of reach of the state-of-the-art and its practical behavior reflects the complexity gain.
\end{abstract}

\section{Categories and Subject Descriptors}

I.1.2 [Computing Methodologies]: Symbolic and Algebraic Manipulation-Algorithms; F.2.2 [Theory of Computation]: Analysis of algorithm and problem complexity-Non numerical algorithms and problems: Geometrical problems and computation

\section{Keywords}

Real dimension; Real solutions; Polynomial systems; Real Geometry.

\section{General Terms}

Algorithms; Theory.

\section{INTRODUCTION}

This paper is devoted to the design and the implementation of an algorithm for computing the real dimension $d$ of an algebraic set $V \cap \mathbb{R}^{n}$ defined by one polynomial equation $f=0$ with rational coefficients and degree $D$. Recall that when $V \cap \mathbb{R}^{n}$ is empty, its real dimension $d$ is -1 by convention, when it is non-empty but finite it is 0 else it is

\footnotetext{
* The authors are supported by the GEOLMI grant (ANR 2011 BS03 011 06) of the French National Research Agency.
}

Permission to make digital or hard copies of all or part of this work for personal or classroom use is granted without fee provided that copies are not made or distributed for profit or commercial advantage and that copies bear this notice and the full citation on the first page. Copyrights for components of this work owned by others than ACM must be honored. Abstracting with credit is permitted. To copy otherwise, or republish, to post on servers or to redistribute to lists, requires prior specific permission and/or a fee. Request permissions from permissions@acm.org.

Copyright 20XX ACM X-XXXXX-XX-X/XX/XX ...\$15.00. the largest integer $d$ such that there is a projection of $V \cap \mathbb{R}^{n}$ over a $d$-dimensional affine subspace of coordinates with a non-empty interior.

Motivations. Computing the real dimension is a question of first importance since it is a basic topological invariant. It encodes the number of independent motions that are allowed on a geometric body or the number of independent parameters that may vary independently. Hence, computing the real dimension of semi-algebraic sets has many applications in engineering sciences (see e.g. [26] and references therein). It also has some algorithmic interest since the knowledge of the real dimension can be exploited to accelerate other algorithms studying real algebraic or semi-algebraic sets (see e.g. [8, Section 13.3] or $[6,7])$.

State-of-the-art. Quantifier elimination (QE) over the reals plays a central role for computing the real dimension since it allows to obtain semi-algebraic descriptions of projections of semi-algebraic sets. Hence, it allows to decide if the interior of such a projection is empty. Consequently, Cylindrical Algebraic Decomposition (CAD) due to Collins [12] can be used for computing the real dimension. However, the arithmetic complexity of this algorithm is doubly exponential in the total number of variables. Several software implementing variants and improvements of CAD have been designed (Mathematica, Maple, QEPCAD, RedLog, etc.) but because of this doubly exponential complexity they are rather limited to 3 or 4 variables on a wide range of examples.

The current algorithms within the best known complexity class are due to Basu, Pollack and Roy [9] (see also [8, Chapter 14]) following previous work of Koiran [28] and Vorobjov [40]. Let $S \subset \mathbb{R}^{n}$ be a real algebraic set defined by a polynomial equation of degree $D$ with rational coefficients.

These algorithms use QE techniques that essentially allow to compute the projection of $S$ on a $i$-dimensional linear subspace in time $D^{O(i(n-i))}$ arithmetic operations [8, Thm 14.16]. Then, the arithmetic complexity of these algorithms is bounded by $D^{O(d(n-d))}$. These algorithms are deterministic and the complexity is output sensitive since it depends on $d$. They also allow to handle general semialgebraic sets in time $(s D)^{O(d(n-d))}$ (where $s$ is the number of inequalities). However several questions remain open.

1. What is the complexity constant hidden in the exponent of the above complexity estimates?

2. Can we obtain an efficient implementation that reflects the complexity gain compared to doubly exponential algorithms?

Main results. We provide answers to both questions: we obtain a probabilistic algorithm whose arithmetic complexity is essentially cubic in $D^{d(n-d)}$; a first implementation shows that it can tackle examples that are out of reach of the state-of-the-art. We give more details on our methodology below.

In the whole paper, let $f \in \mathbb{Q}\left[X_{1}, \ldots, X_{n}\right] \backslash\{0\}$ of degree $D$ and let $V \subset \mathbb{C}^{n}$ be the algebraic set defined by $f=0$. Our technique is still based on the investigation of projections of $V \cap \mathbb{R}^{n}$. Let $\pi_{i}$ 
be the canonical projection $\left(\mathbf{x}_{1}, \ldots, \mathbf{x}_{n}\right) \rightarrow\left(\mathbf{x}_{1}, \ldots, \mathbf{x}_{i}\right)$. Remark that in order to decide if $\pi_{i}\left(V \cap \mathbb{R}^{n}\right)$ has an empty interior, there is no need to compute a semi-algebraic description of this projection using general QE techniques. Indeed, it is sufficient to compute $(i)$ a polynomial that defines a hypersurface containing the boundary of $\pi_{i}\left(V \cap \mathbb{R}^{n}\right)$, (ii) compute sample points in each connected component of the complementary of the real trace of that hypersurface and (iii) for each such sample point $y$, decide if the fiber $\pi_{i}^{-1}(y) \cap V \cap \mathbb{R}^{n}$ is empty. If there is no non-empty real fiber then the interior of $\pi_{i}(V)$ is empty.

This process has already been identified and formalized in [23, 24] where a dedicated projection step has been designed for quantifier elimination over the reals under some conditions on the input. These are regularity conditions (the algebraic set defined by $f=0$ must be smooth) and properness conditions (for any $y \in \mathbb{R}^{i}$, there is a closed ball $B$ containing $y$ such that $\pi_{i}^{-1}(B) \cap V \cap \mathbb{R}^{n}$ is closed and bounded).

Note that when $V$ is smooth, if $V \cap \mathbb{R}^{n} \neq \emptyset$, then its real dimension is $n-1$ by the implicit function theorem. Thus, in our context, this regularity condition is a strong obstruction since on all examples where the real dimension does not coincide with the complex one, this condition is not satisfied.

Moreover, the properness of the restriction of $\pi_{i}$ to $V \cap \mathbb{R}^{n}$ cannot be always ensured, especially when $V \cap \mathbb{R}^{n}$ is not bounded and $i$ is less than the dimension of $V \cap \mathbb{R}^{n}$.

Hence, results in $[23,24]$ are not sufficient and need to be generalized for our purpose. To do that we investigate polar varieties of a deformation $V_{\varepsilon}$ of $V$ defined by $f-\varepsilon=0$ (where $\varepsilon$ is an infinitesimal encoding a small perturbation of the constant coefficient in $f$ ). This allows us to retrieve a regular situation ( $V_{\varepsilon}$ is actually smooth). Next, we show that properness assumptions in $[23,24]$ can be substituted with properness assumptions on polar varieties of $V_{\varepsilon}$ as in [35].

Our algorithm is probabilistic because its correctness depends on some changes of coordinates that are performed randomly. Indeed, we prove that for such a generic choice these properness assumptions on polar varieties are satisfied. Finally, letting the deformation $\varepsilon$ tend to 0 , this allows us to obtain a hypersurface defining the boundary of $\pi_{i}(V)$. We finally get a routine for deciding the emptiness of the interior of $\pi_{i}\left(V \cap \mathbb{R}^{n}\right)$ in generic coordinates. Also, an extra outcome of the paper is a generalization of several results in $[23,24]$.

These geometric steps can be eliminated using many algebraic elimination routines. To estimate the complexity we mainly rely on [17]. We use the arithmetic complexity model over $\mathbb{Q}$ : we count arithmetic operations over $\mathbb{Q}$ as a unit. Below, $\tilde{O}(x)$ means $O\left(x \log (x)^{a}\right)$ for some $a>0$. We can now state our main result.

THEOREM 1. Let $f$ be a polynomial in $\mathbb{Q}\left[X_{1}, \ldots, X_{n}\right] \backslash\{0\}$ of degree $D$ and let $d$ be the real dimension of the real algebraic set defined by $f=0$. There exists a probabilistic algorithm which computes $d$ in time $\tilde{O}\left(n^{16}(1+D)^{3 d(n-d)+5 n+5}\right)$.

Also, note that when a real algebraic set is defined by a polynomial system $f_{1}=\cdots=f_{p}=0$ with coefficients in $\mathbb{Q}$, our algorithm can be used with input $f_{1}^{2}+\cdots+f_{p}^{2}$.

We also report on the practical performances of an implementation of our algorithm. We have used as benchmarks sums of squares of random dense polynomials, discriminants of characteristic polynomials of linear symmetric matrices and series of polynomials that are known to be non-negative over the reals. For all these polynomials, the dimensions of the real algebraic sets they define may vary. We find that our implementation allows to tackle polynomials that are out of reach of the best CAD implementations. As importantly, we emphasize that, in practice, the behaviour of our implementation is output sensitive. Indeed, for families of fixed dimension, timings seem to show a behaviour of type $D^{O(n)}$ but computations performed better when $d$ or $n-d$ are small w.r.t $n / 2$.

Related works. As already mentioned, algorithms in $[8,28,40]$ are the first ones with a singly exponential complexity for computing the real dimension of semi-algebraic sets.
The use of polar varieties in symbolic computation appears first in [1] to compute sample points in smooth equidimensional real algebraic sets (see also [2, 3, 4, 5] and reference therein). There are also used for global optimization and for computing roadmaps (see [18, 19, 36] and references therein).

Properness properties of the restriction of a projection to a polar variety are introduced in [35] and used in [23, 24].

Our complexity estimates rely on complexity results on the geometric resolution algorithm; we refer to $[17,29,37]$ and references therein for a description of these algorithms and their parametric variants.

\section{Structure of the paper.}

Section 2 is devoted to preliminaries and notation used throughout the paper. Section 3 is devoted to the description of the algorithm, the proof of its correctness and its complexity analysis. Section 4 is devoted to the proof of geometric results on which the algorithm relies. The last section reports on practical experiments.

\section{PRELIMINARIES}

We start with basic notions and some notation on algebraic sets.

Algebraic sets. Let $\mathbb{K}$ be a field of characteristic $0, \overline{\mathbb{K}}$ be its algebraic closure. Let $I$ be an ideal of $\mathbb{K}\left[X_{1}, \ldots, X_{n}\right]$ generated by $\left(g_{1}, \ldots, g_{s}\right)$. The $\mathbb{K}$-algebraic set associated to $I$ is the $\mathbb{K}$-algebraic set defined by the polynomial equations $g_{1}=\cdots=g_{s}=0$; we denote it by $V(I)$.

Let $W \subset \overline{\mathbb{K}}^{n}$ be a $\mathbb{K}$-algebraic set defined by polynomial equations in $\mathbb{K}\left[X_{1}, \ldots, X_{n}\right]$. The dimension of $W$ is defined as the Krull dimension of its associated ideal $I(W)=\left\{g \in \mathbb{K}\left[X_{1}, \ldots, X_{n}\right] \mid \forall x \in\right.$ $W, g(x)=0\}$ (see e.g. [14]). This notion of dimension coincides with other notions inspired by differential or algebraic geometry (see e.g. [14, Part II]). Roughly speaking, it is the number of generic hypersurfaces such that their intersection with $W$ is a finite set of points. If $W^{\prime}$ is another algebraic set and $W \subset W^{\prime}$, then the dimension of $W$ is at most the dimension of $W^{\prime}$.

The $\mathbb{K}$-algebraic set $W$ is said to be $\mathbb{K}$-irreducible if it cannot be decomposed as the union of two different $\mathbb{K}$-algebraic sets. Any $\mathbb{K}$ irreducible algebraic set $W$ is uniquely decomposed as a finite union of $\mathbb{K}$-irreducible algebraic sets; these are called the irreducible components of $W$.

When all the irreducible components of $W$ have the same dimension, we say that $W$ is equidimensional.

Let $W$ be an $\mathbb{K}$-algebraic set, equidimensional of co-dimension $c$ and let $\left(f_{1}, \ldots, f_{p}\right)$ be a set of generators of its associated ideal. A point $x \in W$ is called regular if the Jacobian matrix $\left(\frac{\partial f_{i}}{\partial X_{j}}\right)_{\substack{1 \leq i \leq p \\ 1 \leq j \leq n}}$ associated to $\left(f_{1}, \ldots, f_{p}\right)$ has rank $c$ at $x$. The kernel of this Jacobian matrix at $x$ is the tangent space to $W$ at $x$; we denote it by $T_{x} W$. The points in $W$ that are not regular are singular by definition. An algebraic set with no singular points is smooth.

Algebraic sets are closed sets of the Zariski topology. Let $W \subset \overline{\mathbb{K}}^{n}$. The Zariski closure of $W$ is the smallest algebraic set that contains it; we denote it by $\bar{W}$.

Most of the time, the field $\mathbb{K}$ will be clear from the context and will be omitted in the above terminology.

Fields of Puiseux series. We follow the notation of [8, Chap. 2] to define the field of Puiseux series $\mathbb{K}\langle\varepsilon\rangle=\left\{\Sigma_{i \geq i_{0}} a_{i} \varepsilon^{i / q} \mid a_{i} \in \mathbb{K}, q \in\right.$ $\left.\mathbb{N}^{*}, i_{0} \in \mathbb{Z}\right\}$ where $\varepsilon$ is an infinitesimal over $\mathbb{K}$.

Let $S \subset \mathbb{R}^{n}$ be a semi-algebraic set; it is the real solution set of polynomial equations and inequalities with coefficients in $\mathbb{R}$. We denote by $\operatorname{Ext}(S, \mathbb{K}\langle\varepsilon\rangle)$ the set of solutions of this system in $\mathbb{R}\langle\varepsilon\rangle^{n}$.

We say that $y=\sum_{i \geq i_{0}} a_{i} \varepsilon^{i / q}$ in $\mathbb{K}\langle\varepsilon\rangle$ is bounded over $\mathbb{K}$ if $i_{0} \geq 0$. We say that $\mathbf{y}=\left(y_{1}, \ldots, y_{n}\right) \in \mathbb{K}\langle\varepsilon\rangle^{n}$ is bounded over $\mathbb{K}$ if each coordinate $y_{i}$ is bounded over $\mathbb{K}$. Given a bounded element $y \in \mathbb{K}\langle\varepsilon\rangle$ and $\mathbf{y} \in \mathbb{K}\langle\varepsilon\rangle^{n}$, then $\lim _{\varepsilon \rightarrow 0}(y)$ denotes $a_{0}$ in $\mathbb{K}$ and $\lim _{\varepsilon \rightarrow 0}(\mathbf{y})$ denotes the point $\left(\lim _{\varepsilon \rightarrow 0}\left(y_{1}\right), \ldots, \lim _{\varepsilon \rightarrow 0}\left(y_{n}\right)\right) \in \mathbb{K}^{n}$. Given a subset $A \subset \mathbb{K}\langle\varepsilon\rangle^{n}$, we denote by $\lim _{\varepsilon \rightarrow 0}(A)$ the set

$$
\left\{\lim _{\varepsilon \rightarrow 0}(y) \mid y \in A \text { and } y \text { is bounded. }\right\}
$$


We say that $A$ is bounded over $\mathbb{K}$ if evry point in $A$ is bounded over $\mathbb{K}$. By [8, Prop 2.99] the application $\lim _{\varepsilon \rightarrow 0}$ is a ring homomorphism. We recall the following result in [31, Lemma 3.5] that we will use repeatedly in the sequel.

LEMMA 2. [31, Lemma 3.5] Let $f \in \mathbb{Q}\left[X_{1}, \ldots, X_{n}\right]$ be a nonzero polynomial, let $V_{\varepsilon} \subset \mathbb{C}\langle\varepsilon\rangle^{n}$ be algebraic sets defined by the equation $f-\varepsilon=0$. Then, $V_{\varepsilon}$ is either empty or smooth and equidimensional of codimension 1 .

Projections and Polar varieties. Let $W \subset \overline{\mathbb{K}}^{n}$ be an equidimensional algebraic set and let $\left(f_{1}, \ldots, f_{p}\right)$ be a set of generators of the ideal associated to $W$. We denote by $\pi_{i}$ the canonical projection $\left(x_{1}, \ldots, x_{n}\right) \rightarrow\left(x_{1}, \ldots, x_{i}\right)$ for $1 \leq i \leq n$.

A regular point $x \in W$ is a critical point of the restriction of the projection $\pi_{i}$ to $W$ if $\pi_{i}\left(T_{x} W\right) \neq \overline{\mathbb{K}}^{i}$. These are the regular points of $W$ at which the truncated Jacobian matrix $\left(\frac{\partial f_{j}}{\partial X_{k}}\right)_{\substack{1 \leq j \leq p \\ i+1 \leq k \leq n}}$ is rank defective.

The polar variety associated to $\pi_{i}$ and $W$ is the Zariski closure of the critical locus defined above (we refer to [36, Section 2.1]).

Consider now the polynomial $f \in \mathbb{Q}\left[X_{1}, \ldots, X_{n}\right]$ that is to be given as input to our algorithm and the algebraic set $V \subset \mathbb{C}^{n}$ defined by $f=0$. We denote by $V_{\varepsilon} \subset \mathbb{C}\langle\varepsilon\rangle^{n}$ the algebraic set defined by $f=\varepsilon$. By Lemma 2, it is smooth. We will consider the polar varieties associated to $\pi_{i}$ and $V_{\varepsilon}$. They are defined as the zero set in $\mathbb{C}\langle\varepsilon\rangle^{n}$ of

$$
f-\varepsilon=\frac{\partial f}{\partial X_{i+1}}=\cdots=\frac{\partial f}{\partial X_{n}}=0 .
$$

It will be denoted by $W_{\varepsilon, i}$.

Changes of variables and topological notions. We repeatedly use linear changes of variables and projections in the sequel.

The set of invertible matrices with entries in $\mathbb{K}$ is denoted by $\mathrm{GL}_{n}(\mathbb{K})$. Let $\mathbf{A} \in \mathrm{GL}_{n}(\mathbb{K}), g \in \mathbb{K}\left[X_{1}, \ldots, X_{n}\right]$. We denote by $g^{\mathbf{A}}$ the polynomial $g(\mathbf{A X})$ (with $\mathbf{X}=\left[X_{1}, \ldots, X_{n}\right]$ ). For any set of polynomials $G \subset \mathbb{K}[\mathbf{X}]$, we denote by $G^{\mathbf{A}}$ the set $\left\{g^{\mathbf{A}} \mid g \in G\right\}$.

Let $V \subset \overline{\mathbb{K}}^{n}$ be an algebraic set. We denote by $V^{\mathbf{A}} \subset \overline{\mathbb{K}}^{n}$ the image of $V$ by the map $x \mapsto \mathbf{A}^{-1} x$. This notation is naturally extended to semi-algebraic sets when $\mathbb{K}$ is a real closed field.

Assume that $\mathbb{K}$ is equipped with a Euclidean topology. Let $U$ be a subset of $\mathbb{K}^{n}$, we denote by $\operatorname{lnt}(U)$ the interior of $U$ for the Euclidean topology. We denote by $\operatorname{Bd}(U)$ the Euclidean boundary of $U$ defined as the closure of $U$ without its interior.

The properness of a projection is defined as in [35, Section 1] : A map $\pi: A \subset \mathbb{K}^{n} \rightarrow \mathbb{K}^{i}$ is proper at $y \in \mathbb{K}^{i}$ if there exists a neighborhood $\mathcal{O}$ of $y$ such that $\pi^{-1}(\overline{\mathcal{O}})$ is closed in $\mathbb{K}^{n}$ and bounded over $\mathbb{K}^{n}$, where $\overline{\mathcal{O}}$ denotes the closure of $\mathcal{O}$ for the Euclidean topology. If $\pi$ is proper at every point of $\pi(A)$, we simply say that $\pi$ is proper.

\section{ALGORITHM}

\subsection{Descriptions}

We start with the description of the main subroutines. The two following ones are rather standard.

HasRealSolutions: it takes as input a polynomial equation with rational coefficients. It returns true if there exists at least one real solution and false otherwise (see [32, Theorem 4]).

PointsPerComponents: it takes as input a polynomial inequation $g \neq 0$ with rational coefficients. It returns a set of points meeting each connected component of the semi-algebraic set defined by $g \neq 0$ (see [16, Section 4] and [34]).

We describe now the third subroutine. As sketched in the introduction, we need a subroutine that allows to decide if the projection of some real algebraic set has an empty interior. This third subroutine performs this task under some assumptions.
Let $f \in \mathbb{Q}\left[X_{1}, \ldots, X_{n}\right]$ and $0<i<n$ an integer. Below, for $g \in$ $\mathbb{Q}\left[X_{1}, \ldots, X_{n}\right]$, we denote by $I_{i}(g)$ the ideal $\left\langle\frac{\partial g}{\partial X_{i+1}}, \ldots, \frac{\partial g}{\partial X_{n}}\right\rangle$ : $\left\langle\frac{\partial g}{\partial X_{1}}, \ldots, \frac{\partial g}{\partial X_{i}}\right\rangle^{\infty}+\langle g\rangle$ (see [13, Section 4.4] for the definition of saturated ideals).

HasEmptylnterior:

Input: a polynomial $f \in \mathbb{Q}\left[X_{1}, \ldots, X_{n}\right] \backslash\{0\}$, an integer $i$ such that $0<i<n$ and a matrix $\mathbf{A} \in \mathrm{GL}_{n}(\mathbb{Q})$ s.t. $I_{i}\left(f^{\mathbf{A}}\right) \cap \mathbb{Q}\left[X_{1}, \ldots, X_{i}\right]$ is not empty and $\operatorname{Bd}\left(\pi_{i}\left(V^{\mathbf{A}} \cap \mathbb{R}^{n}\right)\right) \subset V\left(I_{i}\left(f^{\mathbf{A}}\right) \cap \mathbb{Q}\left[X_{1}, \ldots, X_{i}\right]\right)$. Output: true if $\operatorname{lnt}\left(\pi_{i}\left(V^{\mathbf{A}} \cap \mathbb{R}^{n}\right)\right)=\emptyset$, false otherwise.

1. compute $g \neq 0$ in the ideal $I_{i}\left(f^{\mathbf{A}}\right) \cap \mathbb{Q}\left[X_{1}, \ldots, X_{i}\right]$

2. let $L=$ PointsPerComponents $(g \neq 0)$.

3. for $\left(\alpha_{1}, \ldots, \alpha_{i}\right) \in L$ do

(a) let $f_{\alpha}^{\mathbf{A}}=f^{\mathbf{A}}\left(\alpha_{1}, \ldots, \alpha_{i}, X_{i+1}, \ldots, X_{n}\right)$

(b) if HasRealSolutions $\left(f_{\alpha}^{\mathbf{A}}=0\right)=$ true then return false

\section{4. return true}

We now describe our main algorithm that is called RealDimension which takes as input $f \in \mathbb{Q}\left[X_{1}, \ldots, X_{n}\right]$. In the following, $\left\lceil\frac{n}{2}\right\rceil \mathrm{de}-$ notes the first integer greater than $\frac{n}{2}$. The algorithm starts by checking that the real algebraic set defined by $f=0$ has solutions. When this is the case, it chooses randomly a linear change of variables and performs successive calls to HasEmptyInterior.

Algorithm RealDimension:

Input: A polynomial $f \in \mathbb{Q}\left[X_{1}, \ldots, X_{n}\right]$.

Output: The real dimension of $V \cap \mathbb{R}^{n}$.

1. if HasRealSolutions $(f=0)=$ false then return -1

2. choose a random matrix $\mathbf{A} \in \mathrm{GL}_{n}(\mathbb{Q})$

3. for $i=1$ to $\left\lceil\frac{n}{2}\right\rceil$ do

(a) if HasEmptylnterior $(f, i, \mathbf{A})=$ true then return $i-1$

(b) if HasEmptylnterior $(f, n-i, \mathbf{A})=$ false then return $n-i$

\subsection{Correctness}

Correctness of Algorithm RealDimension. The correctness proof of Algorithm RealDimension relies on the following results.

Proposition 3. [27, Section 2] Let $W \subset \mathbb{C}^{n}$ be an algebraic set. Assume that $W \cap \mathbb{R}^{n}$ has real dimension $d>0$. Then there exists a non-empty Zariski open set $\Gamma_{1} \subset \mathrm{GL}_{n}(\mathbb{C})$ such that for any $\mathbf{A} \in \Gamma_{1} \cap \mathrm{GL}_{n}(\mathbb{Q})$ and $0<i \leq d$, $\operatorname{lnt}\left(\pi_{d}\left(W^{\mathbf{A}} \cap \mathbb{R}^{n}\right)\right) \neq \emptyset$.

THEOREM 4. Let $0<i<n$ be an integer. There exists a nonempty Zariski open set $\Gamma_{2} \subset \mathrm{GL}_{n}(\mathbb{C})$ such that for all $\mathbf{A} \in \Gamma_{2} \cap$ $\mathrm{GL}_{n}(\mathbb{Q})$, HasEmptylnterior $(f, i, \mathbf{A})$ returns true if $\operatorname{lnt}\left(\pi_{i}\left(V^{\mathbf{A}} \cap\right.\right.$ $\left.\left.\mathbb{R}^{n}\right)\right)$ is empty and false otherwise.

We can now prove the correctness of Algorithm RealDimension with input $f \in \mathbb{Q}\left[X_{1}, \ldots, X_{n}\right]$. We denote by $d$ the real dimension of the real algebraic set defined by $f=0$. We make the assumption that this set is non-empty (the empty case is correctly handled at Step 1). Hence, we assume that $d \geq 0$.

We make the assumption that the matrix $\mathbf{A}$ chosen at step 2 lies in $\Gamma_{1} \cap \Gamma_{2}$ where $\Gamma_{1}$ and $\Gamma_{2}$ are the non-empty Zariski open subsets of $\mathrm{GL}_{n}(\mathbb{C})$ defined in Proposition 3 and Theorem 4.

When the dimension $d$ is 0 , we enter in the loop at Step 3 and the call to HasEmptylnterior $(f, i, \mathbf{A})$ returns true (since $\pi_{1}\left(V^{\mathbf{A}}\right)$ is a finite set of points) and 0 is the returned value.

Assume now that $d>0$. Since $\mathbf{A} \in \Gamma_{1}$ by assumption, Proposition 3 implies that for any $i \leq d, \operatorname{Int}\left(\pi_{i}\left(V^{\mathbf{A}} \cap \mathbb{R}^{n}\right)\right) \neq \emptyset$. Also, by 
definition of the real dimension of a real algebraic set, for any $i>d$, $\operatorname{lnt}\left(\pi_{i}\left(V^{\mathbf{A}} \cap \mathbb{R}^{n}\right)\right)=\emptyset$ holds.

As long as $i \leq d$ and $n-i>d$, the calls to HasEmptylnterior in Step $3 \mathrm{a}$ and Step $3 \mathrm{~b}$ return respectively false and true and the loop goes on by increasing $i$.

Finally if $d<\frac{n}{2}$ and $i=d+1$, then Step 3a returns $i-1=d$. If $d \geq \frac{n}{2}$ and $i=n-d \leq \frac{n}{2}$, the call to HasEmptyInterior at Step 3a returns false and Step $3 b$ returns $n-i=d$.

Correctness of HasEmptylnterior. Assume for the moment the following proposition (we prove it in Section 4).

PROPOSITION 5. There exists a Zariski open set $\Gamma^{\prime} \subset \mathrm{GL}_{n}(\mathbb{C})$ such that for any $\mathbf{A} \in \Gamma^{\prime} \cap \mathrm{GL}_{n}(\mathbb{Q})$ and for any $1 \leq i<n$, the following holds. Let $I_{i}(f)$ be the ideal defined in Subsection 3.1.

1. Let $x \in V\left(\left\langle\frac{\partial f^{\mathbf{A}}}{\partial X_{i+1}}, \ldots, \frac{\partial f^{\mathbf{A}}}{\partial X_{n}}\right\rangle\right)-V\left(\left\langle\frac{\partial f^{\mathbf{A}}}{\partial X_{1}}, \ldots, \frac{\partial f^{\mathbf{A}}}{\partial X_{i}}\right\rangle\right)$. Then the Jacobian matrix associated to $\left(\frac{\partial f^{\mathbf{A}}}{\partial X_{i+1}}, \ldots, \frac{\partial f^{\mathbf{A}}}{\partial X_{n}}\right)$ at $x$ has maximal rank and $V\left(I_{i}\left(f^{\mathbf{A}}\right)\right)$ is either empty or equidimensional of dimension $i-1$.

2. $\operatorname{Bd}\left(\pi_{i}\left(V^{\mathbf{A}} \cap \mathbb{R}^{n}\right)\right)$ is contained in $\pi_{i}\left(V\left(I_{i}\left(f^{\mathbf{A}}\right)\right) \cap \mathbb{R}^{n}\right)$.

We can now prove Theorem 4. As above, $f \in \mathbb{Q}\left[X_{1}, \ldots, X_{n}\right]$ is the polynomial given in the input and $V \subset \mathbb{C}^{n}$ is the algebraic set defined by $f=0$.

Let $\Gamma^{\prime} \subset \mathrm{GL}_{n}(\mathbb{C})$ be the non-empty Zariski open set defined in Proposition 5. Let $\mathbf{A} \in \Gamma^{\prime} \cap \mathrm{GL}_{n}(\mathbb{Q})$. Then by assertion (2) of Proposition 5, $\operatorname{Bd}\left(\pi_{i}\left(V^{\mathbf{A}} \cap \mathbb{R}^{n}\right)\right) \subset \pi_{i}\left(V\left(I_{i}\left(f^{\mathbf{A}}\right)\right) \cap \mathbb{R}^{n}\right)$.

By the elimination theorem [13], we deduce that $\operatorname{Bd}\left(\pi_{i}\left(V^{\mathbf{A}} \cap \mathbb{R}^{n}\right)\right)$ is contained in $V\left(I_{i}\left(f^{\mathbf{A}}\right) \cap \mathbb{Q}\left[X_{1}, \ldots, X_{i}\right]\right) \cap \mathbb{R}^{i}$. Recall also that assertion (1) of Proposition 5 implies that $V\left(I_{i}\left(f^{\mathbf{A}}\right) \cap \mathbb{Q}\left[X_{1}, \ldots, X_{i}\right]\right)$ has codimension $\geq 1$.

So the set $L$ computed at Step 2 contains at least one point in each connected component of the semi-algebraic set defined by $g \neq 0$. Since $\operatorname{Bd}\left(\pi_{i}\left(V^{\mathbf{A}} \cap \mathbb{R}^{n}\right)\right) \subset \pi_{i}\left(V\left(I_{i}\left(f^{\mathbf{A}}\right)\right) \cap \mathbb{R}^{n}\right), L$ contains at least one point in each connected component of $\mathbb{R}^{i}-\operatorname{Bd}\left(\pi_{i}\left(V^{\mathbf{A}} \cap \mathbb{R}^{n}\right)\right)$. The final step of HasEmptylnterior decides the emptiness of $V^{\mathbf{A}} \cap$ $\mathbb{R}^{n} \cap \pi_{i}^{-1}(\alpha)$ for every point $\alpha \in L$.

If $\operatorname{lnt}\left(\pi_{i}\left(V^{\mathbf{A}} \cap \mathbb{R}^{n}\right)\right)=\emptyset$, then $\pi_{i}\left(V^{\mathbf{A}} \cap \mathbb{R}^{n}\right)$ is a subset of its boundary. Then for all $\alpha \in \mathbb{R}^{i}-\operatorname{Bd}\left(\pi_{i}\left(V^{\mathbf{A}} \cap \mathbb{R}^{n}\right)\right)$, the set $V^{\mathbf{A}} \cap \mathbb{R}^{n} \cap$ $\pi_{i}^{-1}(\alpha)$ is empty and HasEmptylnterior returns true as requested.

If $\operatorname{Int}\left(\pi_{i}\left(V^{\mathbf{A}} \cap \mathbb{R}^{n}\right)\right) \neq \emptyset$, then $\operatorname{lnt}\left(\pi_{i}\left(V^{\mathbf{A}} \cap \mathbb{R}^{n}\right)\right)$ contains at least one connected component of $\mathbb{R}^{i}-\operatorname{Bd}\left(\pi_{i}\left(V^{\mathbf{A}} \cap \mathbb{R}^{n}\right)\right)$. So there exists $\alpha \in L$, such that $\alpha$ lies in this component. We deduce that $\alpha$ lies in the interior of $\pi_{i}\left(V^{\mathbf{A}} \cap \mathbb{R}^{n}\right)$. In other words, we have $\pi_{i}^{-1}(\alpha) \cap V^{\mathbf{A}} \cap$ $\mathbb{R}^{n} \neq \emptyset$ and HasEmptyInterior returns false as requested.

\subsection{Complexity analysis}

Our complexity analysis relies mainly on the use of algebraic elimination routines from [17]. The complexity of these routines depends polynomially on geometric degrees of algebraic sets. We investigate below the degrees of the geometric objects manipulated by our algorithm. In the whole paragraph, $f \in \mathbb{Q}\left[X_{1}, \ldots, X_{n}\right]$ denotes the input of RealDimension; it has degree $D$ and the equation $f=0$ defines the real algebraic set $V \cap \mathbb{R}^{n}$.

Degrees of algebraic sets. Let $W \subset \mathbb{C}^{n}$ be a non-empty irreducible algebraic set. The degree $\operatorname{deg}(W)$ of $W$ is defined in [21, Section 2] as the maximal cardinality of a finite set which is obtained by intersecting $W$ with a linear affine subspace. The degree of a reducible closed set is the sum of the degree of its irreducible components.

The complexity of RealDimension and HasEmptylnterior depends on the degree of the objects under study. Let $i$ be an integer such that $0<i<n$. Let $I_{i}(f)$ be the ideal defined in Subsection 3.1. In this paragraph we analyze the degree of the algebraic sets defined respectively by the ideal $I_{i}(f), I_{i}(f) \cap \mathbb{Q}\left[X_{1}, \ldots, X_{n}\right]$ and $\pi_{i}^{-1}(y) \cap$ $V \cap \mathbb{R}^{n}$ where $y$ is a point returned in Step 2 of HasEmptylnterior.
Lemma 6. Let $D$ be the degree of $f$. Then for $1 \leq i \leq n-1$, the degree of $V\left(I_{i}(f)\right)$ and $\pi_{i}\left(V\left(I_{i}(f)\right)\right)$ is bounded by $D^{n-i+1}$ and for all $\mathbf{x}_{i} \in \mathbb{R}^{i}$, the degree of $V \cap \mathbb{R}^{n} \cap \pi_{i}^{-1}\left(\mathbf{x}_{i}\right)$ is bounded by $D$.

Proof. The degree of $f$ is $D \operatorname{so} \operatorname{deg}(V) \leq D$. We denote by $R_{i}$ and $J_{i}$ the ideals $\left\langle\frac{\partial f}{\partial X_{i+1}}, \ldots, \frac{\partial f}{\partial X_{n}}\right\rangle$ and $R_{i}:\left\langle\frac{\partial f}{\partial X_{1}}, \ldots, \frac{\partial f}{\partial X_{i}}\right\rangle^{\infty}$. By [13, Section 4.4, Thm. 7 and Prop. 10],

$$
V\left(J_{i}\right)=\bigcap_{l=1}^{i} \overline{V\left(R_{i}\right)-V\left(\left\langle\frac{\partial f}{\partial X_{l}}\right\rangle\right)} .
$$

Then the degree of $V\left(J_{i}\right)$ is bounded by the product of the degrees of $V\left(R_{i}\right)-V\left(\left\langle\frac{\partial f}{\partial X_{l}}\right\rangle\right)$, each one bounded by $\operatorname{deg}\left(V\left(R_{i}\right)\right)$.

With Bezout's inequality [21], the degree of $V\left(R_{i}\right)$ is bounded by $\operatorname{deg}\left(\bigcap_{r=i+1}^{n} V\left(\left\langle\frac{\partial f}{\partial X_{r}}\right\rangle\right)\right) \leq D^{n-i}$.

Since $I_{i}(f)=J_{i}+\langle f\rangle$, then the degree of $V\left(I_{i}(f)\right)$ is bounded by $D^{n-i} \cdot D=D^{n-i+1}$ and the bound holds for $\operatorname{deg}\left(\pi_{i}\left(V\left(I_{i}(f)\right)\right)\right)$. Finally, the degree of a fiber above a point $P=\left(x_{1}, \ldots, x_{i}\right)$ is the degree of $V\left(\left\langle f, X_{1}-x_{1}, \ldots, X_{i}-x_{i}\right\rangle\right)$ which is $D$.

Complexity estimates. Our goal is to establish the following result.

THEOREM 7. We assume that Algorithm RealDimension chooses $\mathbf{A}$ in $\Gamma_{1} \cap \Gamma_{2} \cap \mathrm{GL}_{n}(\mathbb{Q})$ where $\Gamma_{1}$ and $\Gamma_{2}$ are the non-empty Zariski open subsets of $\mathrm{GL}_{n}(\mathbb{C})$ defined in Proposition 3 and Theorem 4. Let $d$ be the real dimension of $V \cap \mathbb{R}^{n}$, then the number of arithmetic operations needed to compute $d$ is bounded by

$$
\tilde{O}\left(n^{16}(1+D)^{3 d(n-d)+5 n+5}\right) \text {. }
$$

Proof. In the sequel, we omit superscripts $\mathbf{A}$ indicating the changes of variables to keep notation simple. Also, the extra cost induced by these changes of variables are negligible compared to the cost of all other steps of the algorithm.

We start by estimating the complexity of HasEmptylnterior $(f, i, \mathrm{Id})$ for an integer $0<i<n$.

Step 1. By Proposition $5, I_{i}(f)$ has dimension $i-1$ at most. We deduce that $I_{i}(f) \cap \mathbb{Q}\left[X_{1}, \ldots, X_{i}\right]$ is the intersection of ideals $\tilde{I}_{k}$ such that $\tilde{I}_{k} \cap \mathbb{Q}\left[X_{1}, \ldots, X_{i}\right]$ has co-dimension $k$ for $1 \leq k \leq i$. Below, we show how to compute $g_{k} \in \tilde{I}_{k} \cap \mathbb{Q}\left[X_{1}, \ldots, X_{i}\right]$ with $\operatorname{deg}\left(g_{k}\right) \leq$ $\operatorname{deg}\left(V\left(\tilde{I}_{k}\right)\right)$. Since $\operatorname{deg}\left(V\left(I_{i}(f)\right)\right)=\sum_{k=1}^{i} \operatorname{deg}\left(V\left(\tilde{I}_{k}\right)\right)$, we deduce that $g=g_{1} \cdots g_{i}$ has degree less than or equal to $D^{n-i+1}$ (Lemma 6). By Proposition 5, the Jacobian matrix associated to $\left(\frac{\partial f}{\partial X_{i+1}}, \ldots, \frac{\partial f}{\partial X_{n}}\right)$ at $x$ has maximal rank at any point of $W=$ $V\left(\left\langle\frac{\partial f}{\partial X_{i+1}}, \ldots, \frac{\partial f}{\partial X_{n}}\right\rangle\right)-V\left(\left\langle\frac{\partial f}{\partial X_{1}}, \ldots, \frac{\partial f}{\partial X_{i}}\right\rangle\right)$. This implies that we can apply lifting algorithms in [17] at points of the above constructible set. Also note that by [38, Section 6.3], for a generic point $y$ in $\mathbb{C}^{i-1}, V\left(I_{i}(f)\right) \cap \pi_{i-1}^{-1}(y)$ is finite.

One obtains the first polynomial $g_{1}$ in the following way. We first compute generic points in $V\left(I_{i}(f)\right) \cap \pi_{i-1}^{-1}(y)$ where $y$ is a generic point in $\mathbb{Q}^{i-1}$ using [17]; this is possible because Proposition 5 shows that assumptions required in [17] are satisfied. Next, we project those points on the $X_{i}$-coordinate (see e.g. [36, Lemma 10.5.5]) and repeat the process as many times as necessary to perform a multivariate interpolation. Since $g_{1}$ has degree $D^{n-i+1}$, we need $\left(D^{n-i+1}+\right.$ $1)^{i} \leq(D+1)^{i(n-i)}$ interpolation points. Due to the lack of space, we cannot enter into the details. Combining the complexity estimates in [17] and [36, Chap. 10], we get that this is done in time $\tilde{O}\left(n^{5} D^{i(n-i)+3 n-i+4}\right)$.

We now show how to compute $g_{2}$. Note that, choosing $y \in \mathbb{Q}^{i-2}$ generically, $V\left(I_{i}(f)\right) \cap \pi_{i-2}^{-1}(y)$ has dimension 1 . We start by computing generic points in this set, i.e. its intersection with a hyperplane $H$. This is done in two steps. We first obtain a generic point $W$ and next use the lifting procedure [17, Lemma 3] to get a lifting curve that is finally intersected with the hypersurface defined by $f=0[17$, 
Lemma 16]. Remark that repeating the computation with a different $H$ allows us to select, from these generic points, those who actually lie in $V\left(\tilde{I}_{2}\right)$. Finally we project those points who lie in $V\left(\tilde{I}_{2}\right)$ on the $X_{i-1}$-coordinate using again [36, Lemma 10.5] and use again multivariate interpolation to finally reconstruct $g_{2}$. The cost of this step is done in the same complexity bound as above.

Other polynomials $g_{k}$ are obtained similarly. All in all, there are $O(n)$ such steps to perform at most.

The total cost is $\tilde{O}\left(n^{6} D^{i(n-i)+3 n-i+4}\right)$ and $\operatorname{deg}(g) \leq D^{n-i+1}$.

Step 2. We estimate the complexity to compute a set $L$ of points meeting each connected component of the semi-algebraic set defined by $g \neq 0$ in $\mathbb{R}^{i}$ where $g \in \mathbb{Q}\left[X_{1}, \ldots, X_{i}\right]$ has degree bounded by $D^{n-i+1}$. To compute such a set, we take the projection over $\mathbb{R}^{i}$ of a set of points meeting each connected component of the real algebraic set in $\mathbb{R}^{i+1}$ defined by $g Y-1=0$ (where $Y$ is a new indeterminate). The degree of this set is bounded by $\left(D^{n-i+1}+1\right)^{i+1}$. It is straightforward to see that $g Y-1$ is square-free and the algebraic set it defines is smooth and equidimensional. By [35, Theorem 3], this is done using $\tilde{O}\left(n^{16}\left(D^{n-i+1}+1\right)^{3 i+5}\right) \subset \tilde{O}\left(n^{16}(1+D)^{3(n-i) i+5 n-2 i+5}\right)$ arithmetic operations in $\mathbb{Q}$ at most.

Step 3. By [32, Theorem 4], deciding the emptiness of the real algebraic set defined by $f\left(x_{1}, \ldots, x_{i}, X_{i+1}, \ldots, X_{n}\right)=0$ where $\left(x_{1}, \ldots, x_{i}\right)$ is in $L$ is done using $O\left(\left(n D^{n-i}+n^{4}\right) n^{4} D^{2(n-i+1)}\right)$ arithmetic operations in $\mathbb{Q}$ at most. By [35, Theorem 3], the number of points returned by Step 2 is bounded by $O\left(\left(1+D^{n-i+1}\right)^{i+1}\right)$. We deduce that Step 3 uses $O\left(n^{4}\left(n D^{n}+n^{4}\right)(1+D)^{i(n-i)+3 n-i+3}\right)$ arithmetic operations in $\mathbb{Q}$ at most. Note that this step is negligible compared to the complexity of Step 2.

Finally, HasEmptylnterior $(f, i, \mathrm{Id})$ runs in time

$$
\tilde{O}\left(n^{16}(1+D)^{3 i(n-i)+5 n-2 i+5}\right)
$$

Complexity of RealDimension. By [32, Theorem 4], one can decide the emptiness of $V \cap \mathbb{R}^{n}$ in Step 1 in probabilistic time $O\left(\left(n D^{n}+\right.\right.$ $\left.\left.n^{4}\right) n^{4} D^{2(n+1)}\right)$.

Finally, Step 3 of RealDimension is a loop from 1 to $\left\lceil\frac{n}{2}\right\rceil$ calling HasEmptylnterior with inputs $(f, i, \mathrm{Id})$ and $(f, n-i, \mathrm{Id})$. These calls require respectively at most $\tilde{O}\left(n^{16}(1+D)^{3 i(n-i)+5 n-2 i+5}\right)$ and $\tilde{O}\left(n^{16}(1+D)^{3 i(n-i)+3 n+2 i+5}\right)$ arithmetic operations. Since this loop stops when $i$ or $n-i$ is equal to $d$, the complexity of Step 3 and then of RealDimension is bounded by $\tilde{O}\left(n^{16}(1+D)^{3 d(n-d)+5 n+5}\right)$.

\section{PROOF OF PROPOSITION 5}

Strategy of proof. In Subsection 4.1, we prove the existence of a non-empty Zariski open subset $\Gamma_{3} \subset \mathrm{GL}_{n}(\mathbb{C})$ such that, for any $\mathbf{A} \in$ $\Gamma_{3} \cap \mathrm{GL}_{n}(\mathbb{Q})$, assertion (1) of Proposition 5 holds. In Subsection 4.2, we prove the existence of a non-empty Zariski open subset $\Gamma_{4} \subset$ $\mathrm{GL}_{n}(\mathbb{C})$ such that, for any $\mathbf{A} \in \Gamma_{4} \cap \mathrm{GL}_{n}(\mathbb{Q})$, assertion (2) of Proposition 5 holds. Taking $\Gamma^{\prime}=\Gamma_{3} \cap \Gamma_{4}$ ends the proof.

\subsection{Proof of assertion (1) of Proposition 5}

This proof is widely inspired from [1, Prop. 3].

Consider the map $\Phi_{i}$ defined for every $(y, \mathbf{a})=\left(y,\left(a_{k, l}\right)\right) \in \mathbb{C}^{n} \times$ $\mathbb{C}^{n(n-i)}$ by $\Phi_{i}(y, \mathbf{a})=\left(\sum_{k=1}^{n} a_{k, j} \frac{\partial f}{\partial Y_{k}}(y)\right)_{i+1 \leq j \leq n} \in \mathbb{C}^{n-i}$ and, for $\mathbf{a} \in \mathbb{C}^{n(n-i)}$, its restriction $\Phi_{i, \mathbf{a}}: y \in \mathbb{C}^{n} \rightarrow \Phi_{i}(y, \mathbf{a}) \in \mathbb{C}^{n-i}$. The Jacobian matrix of $\Phi_{i}$ with respect to $Y_{1}, \ldots, Y_{n}, a_{1, i+1}, a_{2, i+1}$, $\ldots, a_{n, n}$ at the point $\alpha=\left(y,\left(a_{k, l}\right)\right)$ is the matrix

$$
\left(\begin{array}{cccccccccc}
* & \cdots & * & \frac{\partial f}{\partial Y_{1}} & \cdots & \frac{\partial f}{\partial Y_{n}} & 0 & \cdots & \cdots & 0 \\
\vdots & & \vdots & 0 & \ldots & 0 & \ddots & 0 & \ldots & 0 \\
* & \cdots & * & 0 & \cdots & \cdots & 0 & \frac{\partial f}{\partial Y_{1}} & \cdots & \frac{\partial f}{\partial Y_{n}}
\end{array}\right)
$$

Let $\mathcal{U}_{i} \subset \mathbb{C}^{n}$ be the Zariski open set defined as the set of points such that at least one of the first $i$ partial derivatives of $f$ does not vanish. Let $\alpha$ be in $(y, a) \in \mathcal{U}_{i} \times \mathbb{C}^{n(n-i)}$. The Jacobian matrix has maximal rank at $\alpha$, since otherwise all the partial derivatives of $f$ vanish at $y$ and since $y$ lies in $\mathcal{U}_{i}$, this is impossible. We deduce that $\alpha$ is a regular point of $\Phi_{i}$, which implies that $\Phi_{i}$ is transversal to the origin 0 . By the Weak Transversality Theorem of Thom-Sard [36, Proposition 4.2.2], there exists a Zariski dense subset $\mathcal{O}_{i} \subset \mathbb{C}^{n(n-i)}$ such that for all $\mathbf{a}=\left(a_{k, l}\right)$ in $\mathcal{O}_{i} \cap \mathbb{Q}^{n(n-i)}$, the map $\Phi_{i, \mathbf{a}}: \mathcal{U}_{i} \rightarrow \mathbb{C}^{n-i}$ is transversal to the origin.

Let $\Gamma_{3}$ be the non-empty Zariski subset of $\mathrm{GL}_{n}(\mathbb{C})$ defined as the set of matrices of $\mathrm{GL}_{n}(\mathbb{C})$ such that the $n-i$ last columns lie in $\mathcal{O}_{i}$. Let $\mathbf{A} \in \Gamma_{3} \cap \mathrm{GL}_{n}(\mathbb{Q})$ and $\mathbf{a} \in \mathcal{O}_{i}$ be the $n-i$ last columns of $\mathbf{A}$. We denote by $\mathcal{J}$ the Jacobian matrix of

$$
\left(\sum_{k=1}^{n} a_{k, i+1} \frac{\partial f}{\partial X_{k}}, \ldots, \sum_{k=1}^{n} a_{k, n} \frac{\partial f}{\partial X_{k}}\right)
$$

Then, by the Jacobian Criterion, for all $x$ in

$$
V\left(\sum_{k=1}^{n} a_{k, i+1} \frac{\partial f}{\partial X_{k}}, \ldots, \sum_{k=1}^{n} a_{k, i+1} \frac{\partial f}{\partial X_{k}}\right)-V\left(\frac{\partial f}{\partial X_{1}}, \ldots, \frac{\partial f}{\partial X_{i}}\right),
$$

the matrix $\mathcal{J}$ has maximal rank at $x$. Since $\frac{\partial f^{\mathbf{A}}}{\partial X_{j}}=\sum_{k=1}^{n} a_{k, n} \frac{\partial f}{\partial X_{k}} \circ$ $\mathbf{A}$, then the Jacobian of $\left(\frac{\partial f^{\mathbf{A}}}{\partial X_{i+1}}, \ldots, \frac{\partial f^{\mathbf{A}}}{\partial X_{n}}\right)$ equals $\mathcal{J} \cdot \mathbf{A}$. Let $y$ be in $V\left(\frac{\partial f^{\mathbf{A}}}{\partial X_{i+1}}, \ldots, \frac{\partial f^{\mathbf{A}}}{\partial X_{n}}\right)-V\left(\left(\frac{\partial f}{\partial X_{1}}\right)^{\mathbf{A}}, \ldots,\left(\frac{\partial f}{\partial X_{i}}\right)^{\mathbf{A}}\right)$. Without loss of generality, we assume that $\frac{\partial f}{\partial X_{1}}(\mathbf{A} y) \neq 0$. We now prove that $y \notin V\left(\frac{\partial f^{\mathbf{A}}}{\partial X_{1}}, \ldots, \frac{\partial f^{\mathbf{A}}}{\partial X_{i}}\right)$. Otherwise, since $\frac{\partial f^{\mathbf{A}}}{\partial X_{j}}=$ $\sum_{k=1}^{n} a_{k, j} \frac{\partial f}{\partial X_{k}} \circ \mathbf{A}$, for every integer $j \leq n, \sum_{k=1}^{n} a_{k, 1} \frac{\partial f}{\partial X_{k}}(\mathbf{A} y)=$ 0 with $\mathbf{A} \in \mathrm{GL}_{n}(\mathbb{Q})$ and $\frac{\partial f}{\partial X_{1}}(\mathbf{A} y) \neq 0$ which is impossible. Let $\mathbf{A} \in \Gamma_{3} \cap \mathrm{GL}_{n}(\mathbb{Q})$ and let $K_{i}$ be the set

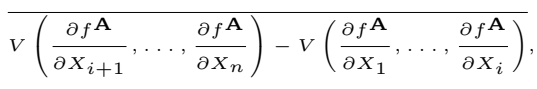

then by the Jacobian Criterion, $K_{i}$ has dimension $i$. So, if $K_{i} \cap V^{\mathbf{A}}$ is non-empty then by Krull's theorem, it has either dimension $i$ or dimension $i-1$. We prove below that $K_{i} \cap V^{\mathbf{A}}$ has dimension $i-1$ or is empty. In the sequel, we omit the superscript $\mathbf{A}$ to keep simple notations. Of course, we assume that $K_{i} \neq \emptyset$ in the sequel.

Let $z \in K_{i}$ be a regular point such that $y \notin V\left(\frac{\partial f}{\partial X_{1}}, \ldots, \frac{\partial f}{\partial X_{i}}\right)$. Without loss of generality, one can assume that $z$ is the origin that we denote by $\mathbf{0}$. Since $K_{i}$ has dimension $i$ and $\mathbf{0}$ is a regular point of $K_{i}$, there exists $\left\{j_{1}, \ldots, j_{i}\right\}$ such that the projection of $T_{z} K_{i}$ on the $\left(X_{j_{1}}, \ldots, X_{j_{i}}\right)$-space is full dimensional (hence the differential of the restriction of the projection to $K_{i}$ at $\mathbf{0}$ is surjective). To keep notations as simple as possible, we assume without loss of generality that $\left\{j_{1}, \ldots, j_{i}\right\}=\{1, \ldots, i\}$.

By the Implicit Function Theorem, there exist two Zariski open sets $\mathcal{U} \subset \mathbb{C}^{i}$ and $\mathcal{V} \subset \mathbb{C}^{n-i}$ and there exists a function

$$
\phi: \mathbf{x}_{i} \in \mathcal{U} \rightarrow\left(\phi_{i+1}\left(\mathbf{x}_{i}\right), \ldots, \phi_{n}\left(\mathbf{x}_{i}\right)\right) \in \mathcal{V}
$$

such that for every $\left(x_{1}, \ldots, x_{n}\right) \in \mathcal{U} \times \mathcal{V}$, the following holds

$$
\left(x_{1}, \ldots, x_{n}\right) \in K_{i} \Leftrightarrow\left(x_{i+1}, \ldots, x_{n}\right)=\left(\phi_{i+1}\left(\mathbf{x}_{i}\right), \ldots, \phi_{n}\left(\mathbf{x}_{i}\right)\right) .
$$

We define now $\gamma$ as the following map: $\mathbf{x}_{i} \in \mathcal{U} \rightarrow\left(\mathbf{x}_{i}, \phi\left(\mathbf{x}_{i}\right)\right) \in$ $\mathcal{U} \times \mathcal{V}$. Remark that any point in the image of $\gamma$ lies in $K_{i}$.

We deduce that for all $\mathbf{x}_{i}=\left(x_{1}, \ldots, x_{i}\right)$,

$$
f \circ \gamma\left(\mathbf{x}_{i}\right)=\sum_{j=1}^{i} \frac{\partial f \circ \gamma}{\partial X_{j}}(\mathbf{0}) x_{j}+o\left(\left\|\mathbf{x}_{i}\right\|\right),
$$

so $f \circ \gamma\left(\mathbf{x}_{i}\right)$ equals to

$$
\sum_{j=1}^{i}\left(\frac{\partial f}{\partial X_{j}}(\mathbf{0})+\sum_{k=i+1}^{n} \frac{\partial f}{\partial X_{k}}(\mathbf{0}) \frac{\partial \phi_{k}}{\partial X_{j}}(\mathbf{0})\right) x_{j}+o\left(\left\|\mathbf{x}_{i}\right\|\right) .
$$


Now, recall that, by definition of $K_{i}, \frac{\partial f}{\partial X_{j}}(\mathbf{0})=0$ for every $j>i$, and that $\mathbf{0} \notin V\left(\frac{\partial f}{\partial X_{1}}, \ldots, \frac{\partial f}{\partial X_{i}}\right)$. Without loss of generality, assume that $\frac{\partial f}{\partial X_{1}}(\mathbf{0}) \neq 0$. Setting $x_{2}=\cdots=x_{i}=0$, we deduce that

$$
f \circ \gamma\left(x_{1}, 0, \ldots, 0\right)=\frac{\partial f}{\partial X_{1}}(\mathbf{0}) x_{1}+o\left(\left\|\mathbf{x}_{i}\right\|\right)
$$

which implies that $f$ is not identically 0 along the curve defined by $\gamma\left(x_{1}, 0, \ldots, 0\right)$ with $\left(x_{1}, 0, \ldots, 0\right) \in \mathcal{U}$. This implies that $K_{i} \cap V$ is either empty or it has dimension $\operatorname{dim}\left(K_{i}\right)-1=i-1$.

\subsection{Proof of assertion (2) of Proposition 5}

It relies on the two following lemmas.

LEMMA 8. There exists a non-empty Zariski open subset $\Gamma_{5} \subset$ $\mathrm{GL}_{n}(\mathbb{C})$ such that for all $\mathbf{A} \in \Gamma_{5} \cap \mathrm{GL}_{n}(\mathbb{Q})$ the following holds. Let $y \in \operatorname{Bd}\left(\pi_{i}\left(V^{\mathbf{A}} \cap \mathbb{R}^{n}\right)\right)$ and let $B_{i} \subset \mathbb{R}^{i}$ be a ball centered at $y$ of radius $r>0$. There exist $x \in V^{\mathbf{A}} \cap \mathbb{R}^{n}$ such that $\pi_{i}(x)=y$, a ball $B_{n} \subset \mathbb{R}^{n}$ centered at $x$ of radius $r^{\prime}>0$ such that $\pi_{i}\left(B_{n}\right) \subset B_{i}$ and $y_{\varepsilon} \in \operatorname{Bd}\left(\pi_{i}\left(\operatorname{Ext}\left(B_{n}, \mathbb{R}\langle\varepsilon\rangle\right) \cap V_{\varepsilon}^{\mathbf{A}}\right)\right)$ such that $\lim _{\varepsilon \rightarrow 0}\left(y_{\varepsilon}\right)=y$.

Recall from Section 2 that $V_{\varepsilon} \in \mathbb{C}\langle\varepsilon\rangle^{n}$ denotes the algebraic set defined by $f-\varepsilon=0$ and that $W_{\varepsilon, i}^{\mathbf{A}} \subset \mathbb{C}\langle\varepsilon\rangle$ denotes the polar variety defined by $f^{\mathbf{A}}-\varepsilon=\frac{\partial f^{\mathbf{A}}}{\partial X_{i+1}}=\cdots=\frac{\partial f^{\mathbf{A}}}{\partial X_{n}}=0$.

LemmA 9. There exists a non-empty Zariski open subset $\Gamma_{6} \subset$ $\mathrm{GL}_{n}(\mathbb{C})$ such that the following holds. For all $\mathbf{A} \in \Gamma_{6} \cap \mathrm{GL}_{n}(\mathbb{Q})$, for any integer $1 \leq i \leq n-1$, the restriction of $\pi_{i-1}$ to the polar variety $W_{\varepsilon, i}^{\mathbf{A}}$ is proper.

Proof of ASSERTION (2) OF PRoposition 5. Let $R_{i}$ and $J_{i}$ be the ideals $\left\langle\frac{\partial f}{\partial X_{i+1}}, \ldots, \frac{\partial f}{\partial X_{n}}\right\rangle$ and $R_{i}:\left\langle\frac{\partial f}{\partial X_{1}}, \ldots, \frac{\partial f}{\partial X_{i}}\right\rangle^{\infty}$. Let $I_{i}(f)$ be the ideal $J_{i}+\langle f\rangle$. First we prove that $\lim _{\varepsilon \rightarrow 0}\left(W_{\varepsilon, i} \cap\right.$ $\left.\mathbb{R}\langle\varepsilon\rangle^{n}\right) \subset V\left(I_{i}(f)\right)$.

Let $\left(x, x_{\varepsilon}\right) \in \lim _{\varepsilon \rightarrow 0}\left(W_{\varepsilon, i} \cap \mathbb{R}\langle\varepsilon\rangle^{n}\right) \times W_{\varepsilon, i}$ such that $\lim _{\varepsilon \rightarrow 0}\left(x_{\varepsilon}\right)=$ $x$. Since $\lim _{\varepsilon \rightarrow 0}$ is a ring homomorphism and $f \in \mathbb{Q}\left[X_{1}, \ldots, X_{n}\right]$, $\lim _{\varepsilon \rightarrow 0}\left(f\left(x_{\varepsilon}\right)\right)=0=f\left(\lim _{\varepsilon \rightarrow 0}\left(x_{\varepsilon}\right)\right)=f(x)$.

Let $h \in J_{i}$; by definition of $J_{i}$, there exists $m \in \mathbb{N}^{*}$ such that for all $g \in\left\langle\frac{\partial f}{\partial X_{1}}, \ldots, \frac{\partial f}{\partial X_{i}}\right\rangle$, the polynomial $g^{m} h$ lies in $R_{i}$. Since $x_{\varepsilon}$ lies in $W_{\varepsilon, i}, g^{m}\left(x_{\varepsilon}\right) h\left(x_{\varepsilon}\right)=0$ for all $g$ in $\left\langle\frac{\partial f}{\partial X_{1}}, \ldots, \frac{\partial f}{\partial X_{i}}\right\rangle$.

Since $V_{\varepsilon}$ is smooth by Lemma 2 , there exists $j \in\{1, \ldots, i\}$ such that $\frac{\partial f}{\partial X_{j}}\left(x_{\varepsilon}\right) \neq 0$. Let $g$ be the polynomial $\frac{\partial f}{\partial X_{j}}$, then $g\left(x_{\varepsilon}\right) \neq 0$ and $g^{m}\left(x_{\varepsilon}\right) h\left(x_{\varepsilon}\right)=0$ so $h\left(x_{\varepsilon}\right)=0$. Finally, since $h$ has rational coefficients and $\lim _{\varepsilon \rightarrow 0}$ is a ring homomorphism, we deduce that $h\left(\lim _{\varepsilon \rightarrow 0}\left(x_{\varepsilon}\right)\right)=h(x)=0$. Then $x$ lies in $V\left(J_{i}+\langle f\rangle\right)=$ $V\left(I_{i}(f)\right)$. We conclude that $\lim _{\varepsilon \rightarrow 0}\left(W_{\varepsilon, i} \cap \mathbb{R}\langle\varepsilon\rangle^{n}\right) \subset V\left(I_{i}(f)\right)$. We claim that there exists a non-empty Zariski open subset $\Gamma_{4} \subset$ $\mathrm{GL}_{n}(\mathbb{C})$ such that for all $\mathbf{A} \in \Gamma_{4} \cap \mathrm{GL}_{n}(\mathbb{Q})$, and for $1 \leq i<n$, $\operatorname{Bd}\left(\pi_{i}\left(V^{\mathbf{A}} \cap \mathbb{R}^{n}\right)\right) \subset \pi_{i}\left(\lim _{\varepsilon \rightarrow 0}\left(W_{\varepsilon, i}^{\mathbf{A}} \cap \mathbb{R}\langle\varepsilon\rangle^{n}\right)\right)$.

Since we already proved $\lim _{\varepsilon \rightarrow 0}\left(W_{\varepsilon, i}^{\mathbf{A}} \cap \mathbb{R}\langle\varepsilon\rangle^{n}\right) \subset V\left(I_{i}^{\mathbf{A}}\right)$, we deduce that $\operatorname{Bd}\left(\pi_{i}\left(V^{\mathbf{A}} \cap \mathbb{R}^{n}\right)\right) \subset \pi_{i}\left(V\left(I_{i}^{\mathbf{A}}\right) \cap \mathbb{R}^{n}\right)$ which ends the proof of assertion (2) of Proposition 5.

Let $\Gamma_{5}$ be the non-empty Zariski open set of $\mathrm{GL}_{n}(\mathbb{C})$ defined in Lemma 8. Let $\Gamma_{6}$ be the non-empty Zariski open set of $\mathrm{GL}_{n}(\mathbb{C})$ defined in Lemma 9. We prove that taking the non-empty Zariski open set $\Gamma_{4}=\Gamma_{5} \cap \Gamma_{6}$ allows to prove our claim.

Let $\mathbf{A} \in \Gamma_{4} \cap \mathrm{GL}_{n}(\mathbb{Q})$. Let $y \in \operatorname{Bd}\left(\pi_{i}\left(V^{\mathbf{A}} \cap \mathbb{R}^{n}\right)\right)$ and $B_{i} \subset \mathbb{R}^{i}$ be the ball centered at $y$ of radius $r>0$. Since $\mathbf{A} \in \Gamma_{5} \cap \mathrm{GL}_{n}(\mathbb{Q})$, there exists $x, B_{n}$ and $y$ as in Lemma 8. In particular, we have $y_{\varepsilon} \in$ $\operatorname{Bd}\left(\pi_{i}\left(\operatorname{Ext}\left(B_{n}, \mathbb{R}\langle\varepsilon\rangle\right) \cap V_{\varepsilon}^{\mathbf{A}}\right)\right)$ such that $\lim _{\varepsilon \rightarrow 0}\left(y_{\varepsilon}\right)=y$.

By Lemma $2, V_{\varepsilon}$ is either empty or smooth and equidimensional. By [35, Proposition 4], the set $\operatorname{Bd}\left(\pi_{i}\left(V_{\varepsilon}^{\mathbf{A}} \cap \mathbb{R}\langle\varepsilon\rangle^{n}\right)\right)$ is a subset of $\pi_{i}\left(W_{\varepsilon, i}^{\mathbf{A}} \cap \mathbb{R}\langle\varepsilon\rangle^{n}\right)$, so $\operatorname{Bd}\left(\pi_{i}\left(\operatorname{Ext}\left(B_{n}, \mathbb{R}\langle\varepsilon\rangle\right) \cap V_{\varepsilon}^{\mathbf{A}}\right)\right)$ is a subset of $\pi_{i}\left(\operatorname{Ext}\left(B_{n}, \mathbb{R}\langle\varepsilon\rangle\right) \cap W_{\varepsilon, i}^{\mathbf{A}}\right)$. By Lemma 9, since $\mathbf{A} \in \Gamma_{6} \cap$
$\mathrm{GL}_{n}(\mathbb{Q})$, the restriction of $\pi_{i}$ to $W_{\varepsilon, i}^{\mathbf{A}}$ is proper. We deduce that $\operatorname{Ext}\left(B_{n}, \mathbb{R}\langle\varepsilon\rangle\right)$ contains a neighborhood $\mathcal{O}$ of $y_{\varepsilon}$ such that $\pi_{i}^{-1}(\overline{\mathcal{O}})$ is closed and bounded. In particular $\pi_{i}^{-1}\left(y_{\varepsilon}\right) \cap \operatorname{Ext}\left(B_{n}, \mathbb{R}\langle\varepsilon\rangle\right) \cap W_{\varepsilon, i}^{\mathbf{A}}$ is not empty. We let $x_{\varepsilon} \in \operatorname{Ext}\left(B_{n}, \mathbb{R}\langle\varepsilon\rangle\right) \cap \pi_{i}^{-1}\left(y_{\varepsilon}\right) \cap W_{\varepsilon, i}^{\mathbf{A}}$. Note that $x_{\varepsilon} \in \operatorname{Ext}\left(B_{n}, \mathbb{R}\langle\varepsilon\rangle\right)$ so it is bounded over $\mathbb{R}$; hence $x^{\prime}=$ $\lim _{\varepsilon \rightarrow 0}\left(x_{\varepsilon}\right)$ exists and $x^{\prime} \in \lim _{\varepsilon \rightarrow 0}\left(\operatorname{Ext}\left(B_{n}, \mathbb{R}\langle\varepsilon\rangle\right) \cap W_{\varepsilon, i}^{\mathbf{A}}\right)=$ $B_{n} \cap \lim _{\varepsilon \rightarrow 0}\left(W_{\varepsilon, i}^{\mathbf{A}}\right)$.

We finish by proving that $\pi_{i}\left(x^{\prime}\right)=y$. We denote by $\left(x_{\varepsilon, 1}, \ldots, x_{\varepsilon, n}\right)$ the coordinates of $x_{\varepsilon}$. Then $\pi_{i}\left(x^{\prime}\right)$ is the point $\pi_{i}\left(\lim _{\varepsilon \rightarrow 0}\left(x_{\varepsilon}\right)\right)=$ $\left(\lim _{\varepsilon \rightarrow 0}\left(x_{\varepsilon, 1}\right), \ldots, \lim _{\varepsilon \rightarrow 0}\left(x_{\varepsilon, i}\right)\right)$. So

$$
\pi_{i}\left(x^{\prime}\right)=\lim _{\varepsilon \rightarrow 0}\left(\left(x_{\varepsilon, 1}, \ldots, x_{\varepsilon, i}\right)\right)=\lim _{\varepsilon \rightarrow 0}\left(\pi_{i}\left(x_{\varepsilon}\right)\right)
$$

which is equal to $\lim _{\varepsilon \rightarrow 0}\left(y_{\varepsilon}\right)=y$.

Finally, we conclude that $\operatorname{Bd}\left(\pi_{i}\left(V^{\mathbf{A}} \cap \mathbb{R}^{n}\right)\right) \subset \pi_{i}\left(\lim _{\varepsilon \rightarrow 0}\left(W_{\varepsilon, i}^{\mathbf{A}}\right) \cap\right.$ $\left.B_{n}\right) \subset \pi_{i}\left(\lim _{\varepsilon \rightarrow 0}\left(W_{\varepsilon, i}^{\mathbf{A}} \cap \mathbb{R}\langle\varepsilon\rangle^{n}\right)\right)$ as requested

Proof of Lemma 8. By [22, Proposition 3], there exists a non-empty Zariski open set $\Gamma_{5} \subset \mathrm{GL}_{n}(\mathbb{C})$ such that for all $\mathbf{A} \in \Gamma_{5} \cap \mathrm{GL}_{n}(\mathbb{Q})$ the following holds. For $1 \leq i \leq n-1$ and for any connected component $C$ of $V^{\mathbf{A}} \cap \mathbb{R}^{n}, \pi_{i}(C)$ is closed. We prove now that $\Gamma_{5}$ satisfies Lemma 8. Let $\mathbf{A}$ be in $\Gamma_{5} \cap \mathrm{GL}_{n}(\mathbb{Q})$.

Assume for the moment the following assertions. (they are proved below).

1. There exists $x \in \pi_{i}^{-1}(y) \cap V^{\mathbf{A}} \cap \mathbb{R}^{n}$ such that the ball $B_{n} \subset$ $\mathbb{R}^{n}$ centered at $x$ of radius $r^{\prime}>0$ satisfies $\pi_{i}\left(B_{n}\right) \subset B_{i}$.

2. The ball $\operatorname{Ext}\left(B_{i}, \mathbb{R}\langle\varepsilon\rangle\right)$ meets $\operatorname{Bd}\left(\pi_{i}\left(\operatorname{Ext}\left(B_{n}, \varepsilon\right) \cap V_{\varepsilon}^{\mathbf{A}}\right)\right)$.

Let $x \in V^{\mathbf{A}} \cap \mathbb{R}^{n}$ and $B_{n}$ be a ball of $\mathbb{R}^{n}$ as in assertion 1 . For every radius $r>0$, we denote by $T_{r}$ the set $\pi_{i}\left(\operatorname{Ext}\left(B_{n}, \mathbb{R}\langle\varepsilon\rangle\right) \cap V_{\varepsilon}^{\mathbf{A}}\right)$. Then by assertion 2 , the set $\operatorname{Bd}\left(T_{r}\right) \cap \operatorname{Ext}\left(B_{i}, \mathbb{R}\langle\varepsilon\rangle\right)$ is non-empty. It is a closed set as it is the intersections of closed sets. It is bounded over $\mathbb{R}$ since it is subset of $\operatorname{Ext}\left(B_{i}, \mathbb{R}\langle\varepsilon\rangle\right)$. We now consider the set $T$ defined by $\bigcap_{r>0} \operatorname{Bd}\left(T_{r}\right) \cap \operatorname{Ext}\left(B_{i}, \mathbb{R}\langle\varepsilon\rangle\right)$. Again, the set $T$ is closed and bounded over $\mathbb{R}$. When $T$ is non-empty, there exists $y_{\varepsilon} \in T$, bounded over $\mathbb{R}$ that belongs to $\operatorname{Ext}\left(B_{i}, \mathbb{R}\langle\varepsilon\rangle\right)$ for any $r>0$. Then $\lim _{\varepsilon \rightarrow 0}\left(y_{\varepsilon}\right)$ exists and equals $y$.

Now, we prove by contradiction that $T$ is non-empty. Fix $r>0$ and let $\eta$ be the distance between $y$ and $\operatorname{Bd}\left(T_{r}\right)$ (defined in [8, Section 3.1]). There are two possible cases: either the distance $\eta$ is 0 or infinitesimally small (i.e $\eta \in \mathbb{R}\langle\varepsilon\rangle$, such that $0<\eta<s$, for all $s \in \mathbb{R}$ ) or there exists $s^{\prime}>0$, with $s^{\prime} \in \mathbb{R}$ such that $\eta>s^{\prime}>0$.

In the first case, for all $s>0$, with $s \in \mathbb{R}$, we have $0 \leq \eta<s$. Since $\eta$ is the distance between $y$ and $\operatorname{Bd}\left(T_{r}\right)$, there exists $y_{\varepsilon} \in T_{r}$ such that the distance $\eta^{\prime}$ between $y$ and $y_{\varepsilon}$ satisfies $\eta \leq \eta^{\prime} \leq \eta+\varepsilon$. Since $\eta+\varepsilon$ is infinitesimal, then for all $s \in \mathbb{R}$ and $s>0,0<\eta+\varepsilon<s$ and $y_{\varepsilon}$ lies in the ball $\operatorname{Ext}\left(B_{i}, \mathbb{R}\langle\varepsilon\rangle\right)$ of radius $s>0$ and $s \in \mathbb{R}$. We deduce that $y_{\varepsilon} \in T$ which contradicts our assumption.

Assume now we are in the second case. By assertion 2 there exists $y_{\varepsilon} \in \operatorname{Bd}\left(T_{s^{\prime}}\right) \cap \operatorname{Ext}\left(B_{i}^{\prime}, \mathbb{R}\langle\varepsilon\rangle\right)$, where $B_{i}^{\prime}$ is the ball centered at $y$ of radius $s^{\prime}$. So the distance $\eta^{\prime}$ between $y$ and $y_{\varepsilon}$ satisfies $\eta \leq \eta^{\prime} \leq$ $s^{\prime}<\eta$ and there is a contradiction.

Finally, the two cases contradict the fact that $T$ is empty.

It remains the prove the above assertions.

1. Since $y$ is in the boundary of $\pi_{i}\left(V^{\mathbf{A}} \cap \mathbb{R}^{n}\right)$, the ball $B_{i}$ meets $\pi_{i}\left(V^{\mathbf{A}} \cap \mathbb{R}^{n}\right)$. We deduce that there exists a connected component $C$ of $V^{\mathbf{A}} \cap \mathbb{R}^{n}$ such that $\pi_{i}(C)$ meets $B_{i}$. Since $\mathbf{A}$ is in $\Gamma_{5} \cap \mathrm{GL}_{n}(\mathbb{Q})$, $\pi_{i}(C)$ is closed. So $y$ is in the boundary of $\pi_{i}(C)$ which is a subset of $\pi_{i}(C)$. We consider a point $x \in \pi_{i}^{-1}(y) \cap C$. Let $r^{\prime}>0$ be such that the ball $B_{n}$ of $\mathbb{R}^{n}$ centered at $x$ of radius $r^{\prime}$, satisfies $\pi_{i}\left(B_{n}\right) \subset B_{i}$. Then $\pi_{i}^{-1}(y) \cap B_{n}$ is not empty.

Without loss of generality, we assume that for any $r^{\prime}$, there exists a point of $B_{n}$ at which $f$ is positive (else we change $f$ to $-f$ ).

2. Assume for the moment the following 
(a) There exists $y_{\varepsilon}$ in $\operatorname{Ext}\left(B_{i}, \mathbb{R}\langle\varepsilon\rangle\right)$ such that

$$
\operatorname{Ext}\left(\pi_{i}^{-1}\left(y_{\varepsilon}\right) \cap B_{n}, \mathbb{R}\langle\varepsilon\rangle\right) \cap V_{\varepsilon}^{\mathbf{A}} \neq \emptyset .
$$

(b) There exists $y^{\prime}$ in $B_{i}$ such that

$$
\operatorname{Ext}\left(\pi_{i}^{-1}\left(y^{\prime}\right) \cap B_{n}, \mathbb{R}\langle\varepsilon\rangle\right) \cap V_{\varepsilon}^{\mathbf{A}}=\emptyset .
$$

On the one hand, since $\operatorname{Ext}\left(\pi_{i}^{-1}\left(y_{\varepsilon}\right) \cap B_{n}, \mathbb{R}\langle\varepsilon\rangle\right) \cap V_{\varepsilon}^{\mathbf{A}} \neq \emptyset$ by assertion (a) and $\pi_{i}\left(B_{n}\right) \subset B_{i}$ by assertion (1), we have

$$
\operatorname{Ext}\left(B_{i}, \mathbb{R}\langle\varepsilon\rangle\right) \cap \pi_{i}\left(\operatorname{Ext}\left(B_{n}, \mathbb{R}\langle\varepsilon\rangle\right) \cap V_{\varepsilon}^{\mathbf{A}}\right) \neq \emptyset .
$$

On the other hand, let $U$ be the complementary of $\operatorname{Ext}\left(B_{n}, \mathbb{R}\langle\varepsilon\rangle\right) \cap$ $V_{\varepsilon}^{\mathbf{A}}$ in $\mathbb{R}\langle\varepsilon\rangle^{n}$. Since $\operatorname{Ext}\left(\pi_{i}^{-1}\left(y^{\prime}\right) \cap B_{n}, \mathbb{R}\langle\varepsilon\rangle\right) \cap V_{\varepsilon}^{\mathbf{A}}=\emptyset$ by assertion (b) and since $\operatorname{Ext}\left(\pi_{i}^{-1}\left(y^{\prime}\right), \mathbb{R}\langle\varepsilon\rangle\right) \neq \emptyset$ by assertion (1), we deduce that $\operatorname{Ext}\left(\pi_{i}^{-1}\left(y^{\prime}\right), \mathbb{R}\langle\varepsilon\rangle\right) \cap U \neq \emptyset$. This implies that $\operatorname{Ext}\left(B_{i}, \mathbb{R}\langle\varepsilon\rangle\right) \cap \pi_{i}(U) \neq \emptyset$.

By [8, Prop 5.24], the set $\operatorname{Ext}\left(B_{i}, \mathbb{R}\langle\varepsilon\rangle\right)$ is semi-algebraically connected. It is the disjoint union of $\operatorname{Ext}\left(B_{i}, \mathbb{R}\langle\varepsilon\rangle\right) \cap \pi_{i}(U)$ and

$$
\operatorname{Ext}\left(B_{i}, \mathbb{R}\langle\varepsilon\rangle\right) \cap \pi_{i}\left(\operatorname{Ext}\left(B_{n}, \mathbb{R}\langle\varepsilon\rangle\right) \cap V_{\varepsilon}^{\mathbf{A}}\right)
$$

which are semi-algebraic sets, closed in $\operatorname{Ext}\left(B_{i}, \mathbb{R}\langle\varepsilon\rangle\right)$. So the set $\operatorname{Ext}\left(B_{i}, \mathbb{R}\langle\varepsilon\rangle\right)$ meets the boundary of $\pi_{i}\left(\operatorname{Ext}\left(B_{n}, \mathbb{R}\langle\varepsilon\rangle\right) \cap V_{\varepsilon}^{\mathbf{A}}\right)$.

Finally, we prove (a) and (b).

(a). We prove that there exists a point $x_{\varepsilon}$ in $\operatorname{Ext}\left(B_{n}, \mathbb{R}\langle\varepsilon\rangle\right) \cap V_{\varepsilon}^{\mathbf{A}}$. Since $f$ is not non-positive over $B_{n}$, there exists $x^{\prime}$ in $B_{n}$ such that $f^{\mathbf{A}}\left(x^{\prime}\right)>0$. Since $f^{\mathbf{A}}\left(x^{\prime}\right) \in \mathbb{R}, x \in V^{\mathbf{A}} \cap \mathbb{R}^{n}$ and $\varepsilon$ is an infinitesimal, we deduce that $f^{\mathbf{A}}(x)-\varepsilon<0$ and $f^{\mathbf{A}}\left(x^{\prime}\right)-\varepsilon>0$.

Let $\Psi$ be the polynomial in $\mathbb{R}\langle\varepsilon\rangle[T]$ defined by $\Psi=f^{\mathbf{A}}(T x+(1-$ $\left.T) x^{\prime}\right)-\varepsilon$. Then $\Psi(0)>0$ and $\Psi(1)<0$ so by [8, Thm 2.11], there exists $t_{0} \in(0,1)$ such that $\Psi\left(t_{0}\right)=0$. Let $x_{\varepsilon}$ be the point $t_{0} x+\left(1-t_{0}\right) x^{\prime} \in \operatorname{Ext}\left(B_{n}, \mathbb{R}\langle\varepsilon\rangle\right)$, then $f^{\mathbf{A}}\left(x_{\varepsilon}\right)-\varepsilon=0$. Then $x_{\varepsilon}$ is in $\operatorname{Ext}\left(B_{n}, \mathbb{R}\langle\varepsilon\rangle\right) \cap V_{\varepsilon}^{\mathbf{A}}$.

Let $y_{\varepsilon}$ be $\pi_{i}\left(x_{\varepsilon}\right)$, then $y_{\varepsilon} \in \pi_{i}\left(\operatorname{Ext}\left(B_{n}, \mathbb{R}\langle\varepsilon\rangle\right)\right) \subset \operatorname{Ext}\left(B_{i}, \mathbb{R}\langle\varepsilon\rangle\right)$. Then $x_{\varepsilon}$ is in $\operatorname{Ext}\left(\pi_{i}^{-1}\left(y_{\varepsilon}\right) \cap B_{n}, \mathbb{R}\langle\varepsilon\rangle\right) \cap V_{\varepsilon}^{\mathbf{A}}$, so there exists $y_{\varepsilon}$ in $\operatorname{Ext}\left(B_{i}, \mathbb{R}\langle\varepsilon\rangle\right)$ such that $\operatorname{Ext}\left(\pi_{i}^{-1}\left(y_{\varepsilon}\right) \cap B_{n}, \mathbb{R}\langle\varepsilon\rangle\right) \cap V_{\varepsilon}^{\mathbf{A}} \neq \emptyset$.

(b). Since $y$ is in $\operatorname{Bd}\left(\pi_{i}\left(V^{\mathbf{A}} \cap \mathbb{R}^{n}\right)\right)$ by assumption and since $\pi_{i}\left(B_{n}\right)$ is a neighborhood of $y$, there exists $y^{\prime} \in \pi_{i}\left(B_{n}\right)$ such that $y^{\prime}$ is not in the Euclidean closure of $\pi_{i}\left(V^{\mathbf{A}} \cap \mathbb{R}^{n}\right)$. We deduce that the distance between $y^{\prime}$ and $\pi_{i}\left(V^{\mathbf{A}} \cap \mathbb{R}^{n}\right)$ is positive. We also deduce that the set $F=\pi_{i}^{-1}\left(y^{\prime}\right) \cap B_{n}$ is not empty.

The distance $\Delta$ between $F$ and $V \cap \mathbb{R}^{n}$ is also positive. Indeed, otherwise, for all $\beta>0$ there is a point $z \in F$ such that the distance between $z$ and $V \cap \mathbb{R}^{n}$ is less than $\beta$. Then $\Delta<\beta$ for all $\beta>0$. This implies that $\Delta=0$ and $F$ meets the closure of $V \cap \mathbb{R}^{n}$ which contradicts the fact that $y^{\prime}$ is not in the closure of the projection.

Since $F \subset B_{n}$ is closed and bounded, then the polynomial function $x \mapsto f(x)$ reach its lower bound $\delta$ at $z_{0} \in F$ and its upper bound $\delta^{\prime}$ at $z_{0}^{\prime} \in F$. Since $\Delta>0$, either $\delta>0$ or $\delta^{\prime}<0$.

We assume now $\delta>0$. Then there exists $\eta>0, \eta \in \mathbb{R}$ such that for all $z^{\prime} \in F, f\left(z^{\prime}\right)>\eta$. If we denote the coordinates of $y^{\prime}$ by $y_{1}^{\prime}, \ldots, y_{i}^{\prime}$, then the semi-algebraic set $F$ can be defined by the equations $X_{1}=y_{1}^{\prime}, \ldots, X_{i}=y_{i}^{\prime}$, the polynomial inequality $d\left(\left(X_{1}, \ldots, X_{n}\right), y\right) \leq r$ and both with or without the inequality $f\left(X_{1}, \ldots, X_{n}\right)>\eta$. Then by [8, Proposition 2.87], for all $z_{\varepsilon} \in$ $\operatorname{Ext}(F, \mathbb{R}\langle\varepsilon\rangle), f\left(z_{\varepsilon}\right)>\eta>0$ with $\eta \in \mathbb{R}$. Thus $f$ never equals to $\varepsilon<\eta$ on $\operatorname{Ext}(F, \mathbb{R}\langle\varepsilon\rangle)=\operatorname{Ext}\left(\pi_{i}^{-1}\left(y^{\prime}\right) \cap B_{n}, \mathbb{R}\langle\varepsilon\rangle\right)$. When $\delta^{\prime}<0$, the proof is similar. We prove that there exists $\eta^{\prime}<0$ such that $f$ is never equals to $\varepsilon>0>\eta^{\prime}>\delta^{\prime}$ when $\delta^{\prime}<0$. In both cases, we deduce that $\operatorname{Ext}\left(\pi_{i}^{-1}\left(y^{\prime}\right) \cap B_{n}, \mathbb{R}\langle\varepsilon\rangle\right) \cap V_{\varepsilon}^{\mathbf{A}}=\emptyset$.

Proof of Lemma 9. This lemma is a generalization to $\mathbb{C}\langle\varepsilon\rangle$ of [35, Theorem 1].

Proof. The proof of [35, Theorem 1] holds if the base field is $\mathbb{C}\langle\varepsilon\rangle$ instead of $\mathbb{C}$. This theorem can be restated as follows: There exists a Zariski open set $\Gamma$ in $\mathrm{GL}_{n}(\mathbb{C}\langle\varepsilon\rangle)$ such that for $\mathbf{A}$ in $\Gamma$ and $i \in\{1, \ldots, n-1\}$, the restriction of $\pi_{i}$ to the $i$-th polar variety $W_{\varepsilon, i}^{\mathbf{A}}$ associated to $f-\varepsilon$ is proper. We now prove that the previous property holds over a non-empty Zariski open subset of $\mathrm{GL}_{n}(\mathbb{C})$.

The proof of [35, Theorem 1] uses $n^{2}$ new indeterminates denoted by $\mathcal{A}_{1,1}, \ldots, \mathcal{A}_{n, n}$, and characterizes the matrices of $\mathrm{GL}_{n}(\mathbb{C}\langle\varepsilon\rangle)$ which do not satisfy the properness property as the set of solutions of a polynomial system $G_{1}=0, \ldots, G_{s}=0$ of $\mathbb{C}\langle\varepsilon\rangle\left[\mathcal{A}_{1,1}, \ldots, \mathcal{A}_{n, n}\right]$. Let $G$ be the product of $G_{1}, \ldots, G_{s}$. The polynomials $G_{1}, \ldots, G_{s}$ are defined as generators of the prime components of the radical of an ideal generated by $f-\varepsilon \in \mathbb{Q}(\varepsilon)\left[X_{1}, \ldots, X_{n}\right]$ and some minors of the Jacobian matrix of $f-\varepsilon$ also in $\mathbb{Q}(\varepsilon)\left[X_{1}, \ldots, X_{n}\right]$ (see $[35$, Sections 2.3 and 2.4]) so their coefficients are in $\mathbb{Q}(\varepsilon)$ and the coefficients of $G$ too. Let $\Omega$ be the non-empty Zariski open set of $\mathrm{GL}_{n}(\mathbb{C}\langle\varepsilon\rangle)$ defined as the complementary of this set of matrices.

If we multiply $G$ by the least common multiple of the denominators of its coefficients, we obtain a polynomial with coefficients in $\mathbb{Q}[\varepsilon]$. Let $P$ be the primitive part of this polynomial. Let $P_{0}$ be the polynomial with rational coefficients obtained by replacing $\varepsilon$ by 0 in $P$, then $P-P_{0}$ can be factorized as $P-P_{0}=\varepsilon^{\nu} P_{\varepsilon}$ with $P_{\varepsilon}$ with coefficients in $\mathbb{Q}[\varepsilon]$ and $\nu>0$ as large as possible. Hence, $P=P_{0}+\varepsilon^{\nu} P_{\varepsilon}$.

Since the coefficients of $P$ have non-trivial gcd, at least one of the two polynomials $P_{0}$ and $P_{\varepsilon}$ is not identically 0 . Indeed, if $P_{0}=0$, then since $\nu$ is maximal, $P=\varepsilon^{\nu} P_{\varepsilon}=\varepsilon^{\nu}\left(P_{1}+\varepsilon^{\nu^{\prime}} P_{2}\right)$ with $P_{1}$ with coefficients in $\mathbb{Q}$ and then $P_{1} \neq 0$. Let $\Omega_{0} \subset \mathrm{GL}_{n}(\mathbb{C})$ (resp. $\Omega_{1} \subset$ $\mathrm{GL}_{n}(\mathbb{C})$ ) be the non-empty Zariski open set defined by $P_{0} \neq 0$ (resp. $\left.P_{1} \neq 0\right)$.

Let $\mathbf{A}$ be in $\left(\Omega_{0} \cup \Omega_{1}\right) \cap \mathrm{GL}_{n}(\mathbb{Q}) \subset \Omega$, then $P(\mathbf{A})=P_{0}(\mathbf{A})+$ $\varepsilon^{\nu} P_{\varepsilon}(\mathbf{A}) \in \mathbb{Q}[\varepsilon]$. We now prove that $P(\mathbf{A}) \neq 0$. If $P_{0}(\mathbf{A})=0$, then $P_{\varepsilon}(\mathbf{A}) \neq 0$ and then $P(\mathbf{A})=\varepsilon^{\nu} P_{\varepsilon}(\mathbf{A}) \neq 0$. If $P_{0}(\mathbf{A}) \neq 0$, then, since $\varepsilon$ is transcendental, $P(\mathbf{A}) \neq 0$. Then $P(\mathbf{A}) \neq 0$ so the restriction of $\pi_{i}$ to $W_{\varepsilon, i}^{\mathbf{A}}$ is proper. Let $\Gamma$ be the set $\Omega_{0} \cup \Omega_{1}$, then for all matrices $\mathbf{A}$ in $\Gamma \cap \mathrm{GL}_{n}(\mathbb{Q})$, the properness property holds.

\section{EXPERIMENTS}

We report on timings obtained with a first implementation of our algorithm. This is a Maple implementation built-on the RAGlib Maple package [33] and the FGb library [15] written in C by J.-C. Faugère. RAGlib is used for deciding the emptiness of real algebraic sets and computing sample points in each of their connected components. It implements algorithms that essentially run in time $D^{O(n)}$. The library FGb is a state-of-the art library for Gröbner bases computations. We use it for all ideal-theoretic operations required by our algorithm. We also use Gröbner bases computations to check Noether position properties needed for the correctness of the algorithm. This allows us to try sparse linear changes of variables (or avoid them when unnecessary) which is crucial for practical performances.

We established that our algorithm runs in time $D^{O(d(n-d))}$ where $d$ is the dimension of the real algebraic set under study. A first goal is to observe if the implementation has a practical behaviour that reflects this complexity. In other words computations should be "easier" when $d$ or $n-d$ is "small" and harder when $d$ is close to $n / 2$. Another goal is to identify if such an implementation can handle examples that are out of reach of the best implementations of Cylindrical Algebraic Decomposition such as QEPCAD [10], the implementation of CAD in Maple [11] or RedLog [39] among others. We report the timings obtained with the Maple implementation of CAD (other mentioned software behave similarly on our test-suite). While it is natural to compare with CAD since it is the unique other implemented technique, remember that CAD provides much more information than the dimension.

The choice of a test-suite is often subjective. With respect to our goals, we have chosen to run the software on sums of squares of random dense polynomials because this allows us to control the dimension of the real algebraic set and identifies if the implementation reflects the $D^{O(d(n-d))}$ complexity. We also have chosen discriminants of characteristic polynomials of linear symmetric matrices (entries are chosen random dense). These are known to be sums of squares 


\begin{tabular}{|c|c|c|c|c|c|c|c|c|c|}
\hline$n$ & ${ }^{\circ}$ & Degrees & $d$ & CAD & Dim & Step 1 & Step 2 & Step 3 & Fibers \\
\hline $\begin{array}{l}4 \\
4\end{array}$ & $\frac{1}{2}$ & 2,1 & $\begin{array}{l}3 \\
2 \\
2\end{array}$ & $0.8 \mathrm{~s}$ & $\begin{array}{l}4.5 \mathrm{~s} \\
54 \mathrm{~s}\end{array}$ & 11 & $\frac{1}{99 \%}$ & $1 \%$ & $\frac{1}{2}$ \\
\hline & & & & $\infty$ & $\begin{array}{l}54 \mathrm{~s} \\
41 \mathrm{~s}\end{array}$ & $25 \%$ & & & \\
\hline 4 & 4 & $2,1,1,1$ & 0 & $\infty$ & $14 \mathrm{~s}$ & $12 \%$ & $1 \%$ & $87 \%$ & 3 \\
\hline 5 & 1 & 2 & 4 & $0.6 \mathrm{~s}$ & $4 \mathrm{~s}$ & 11 & & & \\
\hline 5 & $\frac{2}{3}$ & $\begin{array}{l}2,1,1 \\
2,1\end{array}$ & 2 & $\begin{array}{l}\infty \\
\infty\end{array}$ & $\begin{array}{l}92 \mathrm{~s} \\
94 \mathrm{~s}\end{array}$ & $6 \%$ & $\begin{array}{l}99.4 \% \\
88 \%\end{array}$ & $6 \%$ & $\begin{array}{l}4 \\
1\end{array}$ \\
\hline 5 & 4 & 2,1,1,1, & 1 & $\infty$ & $54 \mathrm{~s}$ & $7 \%$ & $13 \%$ & $\begin{array}{l}80 \% \\
97 \%\end{array}$ & 4 \\
\hline 6 & 1 & 2 & 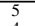 & $7.5 \mathrm{~s}$ & $4.8 \mathrm{~s}$ & 1 & & & 1 \\
\hline & & 2,1 & 4 & $\infty$ & $116 \mathrm{~s}$ & $0 \%$ & $\begin{array}{l}99.8 \% \\
905 \%\end{array}$ & $0.2 \%$ & \\
\hline & & & 2 & & $190 \mathrm{~s}$ & $3 \%$ & $95 \%$ & & \\
\hline 6 & & $2,1,1,1,1$ & & & & & & & \\
\hline & 6 & $2,1,1,1,1,1,1$ & 0 & $\infty$ & $120 \mathrm{~s}$ & $1 \%$ & $0 \%$ & $99 \%$ & 3 \\
\hline $\begin{array}{l}7 \\
7\end{array}$ & $\begin{array}{l}1 \\
2 \\
\end{array}$ & $\begin{array}{l}2 \\
2,1\end{array}$ & $\begin{array}{l}6 \\
5\end{array}$ & $\begin{array}{l}3.1 \mathrm{~s} \\
\infty\end{array}$ & $\begin{array}{l}4.7 \mathrm{~s} \\
222 \mathrm{~s}\end{array}$ & $0 \%$ & $99 \%$ & $1 \%$ & 6 \\
\hline & 3 & $2,1,1$ & 4 & $\infty$ & $2278 \mathrm{~s}$ & $1 \%$ & $96 \%$ & $3 \%$ & 7 \\
\hline & & & 3 & $\infty$ & $103190 \mathrm{~s}$ & & $100 \%$ & & \\
\hline & 6 & $\begin{array}{l}2,1,1,1,1 \\
2,1,1,1\end{array}$ & ${ }_{1}^{2}$ & $\infty_{\infty}^{\infty}$ & 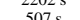 & $2 \%$ & 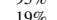 & $79 \%$ & \\
\hline 7 & 7 & $2,1,1,1,1,1,1$ & 0 & $\infty$ & $355 \mathrm{~s}$ & $1 \%$ & $0 \%$ & $99 \%$ & 3 \\
\hline 8 & 1 & 2 & 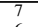 & $24.2 \mathrm{~s}$ & $7.6 \mathrm{~s}$ & $x_{0}$ & & & 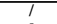 \\
\hline & & & $\frac{0}{5}$ & $\begin{array}{l}\infty \\
\infty\end{array}$ & $\begin{array}{c}2332 \mathrm{~s} \\
\infty\end{array}$ & & $99.5 \%$ & & 8 \\
\hline & 4 & $2,1,1,1$ & 4 & $\infty$ & $\infty$ & & & & \\
\hline & 5 & $2,1,1,1,1,1$ & 3 & $\infty$ & $\infty$ & & & & \\
\hline 8 & 6 & $2,1,1,1,1,1,1$ & ${ }_{2}^{2}$ & $\infty$ & $35377 \mathrm{~s}$ & $1 \%$ & $99 \%$ & $0 \%$ & 1 \\
\hline 8 & 8 & 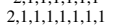 & 0 & $\infty$ & $1002 \mathrm{~s}$ & $0.2 \%$ & $0 \%$ & $99.8 \%$ & 3 \\
\hline 4 & 2 & $2,2,2$ & 2 & $\infty$ & $34 \mathrm{~s}$ & $0.1 \%$ & $97 \%$ & $2.9 \%$ & 8 \\
\hline $\begin{array}{l}4 \\
4 \\
\end{array}$ & $\begin{array}{l}3 \\
4\end{array}$ & $\begin{array}{l}2,2,1 \\
2,2,1,1\end{array}$ & $\begin{array}{l}1 \\
0 \\
0\end{array}$ & $\begin{array}{l}\infty \\
\infty\end{array}$ & $\begin{array}{l}54 \mathrm{~s} \\
25 \mathrm{~s}\end{array}$ & $19 \%$ & $\begin{array}{l}7 \% \\
2 \%\end{array}$ & $79 \%$ & $\begin{array}{l}0 \\
3 \\
\end{array}$ \\
\hline $\begin{array}{l}5 \\
5\end{array}$ & $\begin{array}{c}2 \\
3\end{array}$ & $\begin{array}{l}2,2,1 \\
2,2,1\end{array}$ & $\begin{array}{l}3 \\
2 \\
2\end{array}$ & $\begin{array}{l}\infty \\
\infty \\
\infty\end{array}$ & $\begin{array}{l}79 \mathrm{~s} \\
8003 \mathrm{~s}\end{array}$ & $\begin{array}{l}0 \% \\
3.8 \% \\
\end{array}$ & $\begin{array}{l}99.7 \% \\
96.1 \% \\
\end{array}$ & $.3 \%$ & $\begin{array}{l}1 \\
8 \\
\end{array}$ \\
\hline $\begin{array}{r}5 \\
5\end{array}$ & 4 & $\begin{array}{l}2,2,1,1 \\
2,2,1\end{array}$ & 1 & $\infty$ & $\begin{array}{ll}4773 \mathrm{~s} \\
284 \mathrm{~s}\end{array}$ & $3.1 \%$ & $96.6 \%$ & $0.3 \%$ & 6 \\
\hline 6 & 2 & 2,2 & 4 & $\infty$ & $2477 \mathrm{~s}$ & $0 \%$ & $100 \%$ & $0 \%$ & 1 \\
\hline 6 & & 22,1 & 2 & $\infty$ & $\begin{array}{l}\infty \\
\infty \\
\infty\end{array}$ & & & & \\
\hline 6 & 5 & 1,1 & 1 & $\infty$ & $152500 \mathrm{~s}$ & $3.4 \%$ & $96.6 \%$ & $0 \%$ & 6 \\
\hline 6 & 6 & $2,2,1,1,1,1,1$ & & $\infty$ & $6376 \mathrm{~s}$ & $11 \%$ & $0 \%$ & $89 \%$ & 3 \\
\hline
\end{tabular}

Table 1: Sum of square of $s$ random polynomials in $n$ variables.

[25]. We finally consider sparse polynomials from [20] and [30] that are known to be non-negative over the reals but which have real roots (this implies that the dimension of the real algebraic set they define is less than $n-1$ ).

The computations were performed on an Intel Xeon E7540 @ 2.00 Ghz and 250GB of RAM. All timings are given in seconds. The symbol $\infty$ means that the computation has been stopped after 48 hours. Random dense polynomials. Timings are given in Table 1 . We consider of sums-of-squares of $s n$-variate polynomials. The degrees of the polynomials are given and the computed dimension as well. The column CAD indicates the timing of the Maple CAD implementation. Those for our implementation are given in the column Dim. We also give the relative part of the three main steps of HasEmptylnterior: computation of $I_{i}^{\mathbf{A}} \cap \mathbb{Q}\left[X_{1}, \ldots, X_{i}\right]$, computing sample points outside the zero locus of the obtained polynomial and finally decide the emptiness of fibers; we indicate their number.

It appears that the practical behaviour of our implementation reflects the complexity of our algorithm: for fixed $n$, timings are longer when $d$ approaches $n / 2$ while they are smaller for $d=1$ or $n-1$.

CAD implementations don't have this behaviour and seem to be essentially sensitive to the number of variables. Hence, on this benchmark, our implementation tackles more examples than CAD does.

Discriminant of characteristic polynomials of linear symmetric matrices. For linear matrices of size 2, the associated polynomials have degree 2. Both our implementation and CAD manage to compute the real dimension up to more than 45 variables, and in this specific case the CAD is faster than our implementation. But in Table 2 we see that when the size of the problem increases (through the size of the matrix and hence the input degree), our implementation outperforms CAD implementations when $n>3$. In Table $2, k$ is the size of the matrix, $n$ is the number of variables, $D$ is the degree of the polynomial, and $d$ is the real dimension of the real algebraic set.

Other examples. We finally considered the following polynomials.

1. $g_{n}:=\left(\sum_{i=1}^{n} x_{i}^{2}\right)^{2}-4 \sum_{i=1}^{n-1} x_{i}^{2} x_{i+1}^{2}-4 x_{1}^{2} x_{n}^{2}$, non-negative for $n \geq 4$

2. $f_{n}:=\prod_{i=1}^{n}\left(x_{i}^{2}+n-1\right)-n^{n-2}\left(\sum_{i=1}^{n} x_{i}\right)^{2}$.

Timings are given in Table 5. We observe a similar behaviour than the ones already observed.

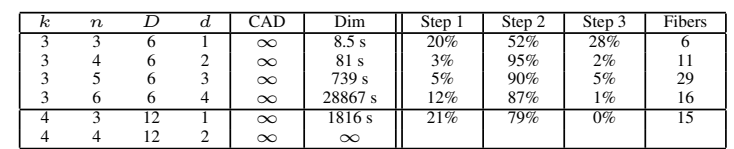

Table 2: Discriminant of characteristic polynomials.

\begin{tabular}{|l|ccc|c|c||c|c|c|c|}
\hline & $n$ & $D$ & $d$ & CAD & Dim & Step 1 & Step 2 & Step 3 & Fibers \\
\hline$g_{3}$ & 3 & 4 & 2 & $0.98 \mathrm{~s}$ & $0.18 \mathrm{~s}$ & $/$ & $/$ & $/$ & $/$ \\
$g_{4}$ & 4 & 4 & 3 & $0.54 \mathrm{~s}$ & $2.1 \mathrm{~s}$ & $/$ & $/$ & $/$ & $/$ \\
$g_{5}$ & 5 & 4 & 2 & $24.6 \mathrm{~s}$ & $42 \mathrm{~s}$ & $3 \%$ & $2 \%$ & $95 \%$ & 14 \\
$g_{6}$ & 6 & 4 & 2 & $\infty$ & $452 \mathrm{~s}$ & $13 \%$ & $43 \%$ & $44 \%$ & 49 \\
$g_{7}$ & 7 & 4 & & $\infty$ & $\infty$ & & & & \\
\hline \hline$f_{3}$ & 3 & 6 & 1 & $2.68 \mathrm{~s}$ & $0.82 \mathrm{~s}$ & $5 \%$ & $30 \%$ & $65 \%$ & 2 \\
$f_{4}$ & 4 & 8 & 1 & $65 \mathrm{~s}$ & $10 \mathrm{~s}$ & $34 \%$ & $2 \%$ & $64 \%$ & 2 \\
$f_{5}$ & 5 & 10 & 1 & $\infty$ & $650 \mathrm{~s}$ & $11 \%$ & $0 \%$ & $89 \%$ & 2 \\
$f_{6}$ & 6 & 12 & 1 & $\infty$ & $\infty$ & & & & \\
\hline
\end{tabular}

Table 3: Series 1 and 2.

\section{REFERENCES}

[1] B. Bank, M. Giusti, J. Heintz, and GM. Mbakop. Polar varieties, real equation solving, and data structures: the hypersurface case. Journal of complexity, 13(1):5-27, 1997.

2] B. Bank, M. Giusti, J. Heintz, and GM. Mbakop. Polar varieties and efficient real elimination. Mathematische Zeitschrift, 238(1):115-144, 2001.

3] B. Bank, M. Giusti, J. Heintz, and L.M. Pardo. Generalized polar varieties and an efficient real elimination.

4] B. Bank, M. Giusti, J. Heintz, and L.M. Pardo. On the intrinsic complexity of point finding in real singular hypersurfaces. Information Processing Leiters, 102(19).1141-1144, 2009.

5] B. Bank, M. Giusti, J. Heintz, M. Safey El Din, and E. Schost. On the geometry of polar varieties. Applicable

6] S. Barone and S. Basu. Refined bounds on the number of connected components of sign conditions on a variety.

[7] S. Barone and S. Basu. On a real analogue of bezout inequality and the number of connected components of sign conditions. ArXiv e-prints, 2013.

8] S. Basu, R. Pollack, and M.-F. Roy. Algorithms in real algebraic geometry, volume 10 of Algorithms and S Basu, R Pollack, and M-F Roy. Computing the dimension of a semi-algebraic set. Journal of Mathematical S Basu, R Pollack, and M-F Roy. Co
Sciences, $134(5): 2346-2353,2006$.

[10] C. Brown, H. Hong, and et al. QEPCAD B - quantifier elimination by partial cylindrical algebraic decomposition http://www.cs.usna.edu/ textasciitildeqepcad/B/QEPCAD.html, 2009.

[11] C. Chen and M. Moreno Maza. Cylindrical algebraic decomposition in the regularchains library. In H. Hong and C. Yap, editors, Mathematical Software - ICMS 2014 - 4th International Congress, Seoul, South Korea, August 5-9, 2014. Proceedings, volume 8592 of Lecture Notes in Computer Science, pages 425-433. Springer, 2014.

[12] G. Collins. Quantifier elimination for real closed fields by cylindrical algebraic decompostion. In Automata Theor and Formal Languages 2 nd GI Conference Kaiserslautern, May 20-23, 1975, pages 134-183. Springer, 1975. D. Cox, J. Little, and D. O'Shea. Ideals, varieties and algorithms: an introduction to computational algebraic
geometry and commutative algebra. Undergraduate Texts in Mathematics. Springer-Verlag, New York, 1997 . geometry and commutative algebra. Undergraduate Texts in Mathematics. Springer-Verlag, New York, 1997.

14] D. Eisenbud. Commutative algebra with a view toward algebraic geometry, volume 27. Springer New York, 1995

[16] J.-C. Faugère, G. Moroz, F. Rouillier, and M. Safey EI Din. Classification of the perspective-three-point problem, J.-C. Faugère, G. Moroz, F. Rouillier, and M. Safey EI Din. Classification of the perspective-three-point pro
discriminant variety and real solving polynomial systems of inequalities. In Proceedings of the twenty-first
international symposium on Symbolic and algebraic computation, pages $79-86$. ACM, 2008.

[17] M. Giusti, G. Lecerf, and B. Salvy. A gröbner free alternative for polynomial system solving. Journal of Complexity, 17(1):154-211, 2001

[18] A. Greuet and M. Safey El Din. Deciding reachability of the infimum of a multivariate polynomial. In Proceedings of the 36th international symposium on Symbolic and algebraic computation, pages 131-138. ACM, 2011.

[19] A. Greuet and M. Safey El Din. Probabilistic algorithm for polynomial optimization over a real algebraic set. SIAM Journal on Optimization, 24(3):1313-1343, 2014.

[20] J. Han, L. Dai, and B. Xia. Constructing fewer open cells by ged computation in cad projection. arXiv preprint arXiv: $1401.4953,2014$

[21] J. Heintz and C.-P. Schnorr. Testing polynomials which are easy to compute. In Proceedings of the twelfth annual ACM symposium on Theory of computing, pages 262-272. ACM, 1980.

[22] D. Henrion, S. Naldi, and M. Safey El Din. Real root finding of determinants of linear matrices. pre-print, 2014. H. Hong and M. Safey El Din. Variant real quantifier elimination: algorithm and application. In Proceedings of the

[24] H. Hong and M. Safey El Din. Variant quantifier elimination. Journal of Symbolic Computation, 47(7):883-901,

[25] N. V. Ilyushechkin. On some identities for the elements of a symmetric matrix. Zapiski Nauchnykh Seminarov POMI, Q. Jin and T. Yang. Overconstraint analysis on spatial 6-link loops. Mechanism and machine theory, 37(3):267-278,

[27] P. Koiran. Randomized and deterministic algorithms for the dimension of algebraic varieties. In Foundations of
Computer Science, 1997. Proceedings., $38 t h$ Annual Symposium on, pages 36-45. IEEE, 1997. P. Koiran. The real dimension problem is npr-complete. Journal of Complexity, 15(2):227 - 238, 1999

[29] G. Lecerf. Computing the equidimensional decomposition of an algebraic closed set by means of lifting fibers. Journal of Complexity, 19(4):564-596, 2003.

[30] P. A. Parrilo. Structured semidefinite programs and semialgebraic geometry methods in robustness and optimization. $\mathrm{PhD}$ thesis, Citeseer, 2000

[31] F. Rouillier, M.-F. Roy, and M. Safey El Din. Finding at least one point in each connected component of a real algebraic set defined by a single equation. Journal of Complexity, 16(4):716-750, 2000 .

2] M. Safey El Din. Finding sampling points on real hypersurfaces is easier in singular situations. MEGA (Effective Methods in Algebraic Geometry) Electronic proceedings, 2005.

[33] M. Safey El Din. RAGlib : Real algebraic geometry library. www-polsys.lip6.fr/ safey/RAGLib/, 2007. 4] M. Safey El Din. Testing sign conditions on a multivariate polynomial and applications, mathematics in computer science journal, special inaugural issue on algorithms and

[35] M. Safey El Din and É. Schost. Polar varieties and computation of one point in each connected component of a smooth real algebraic set. In Proceedings of the 2003 international symposium on Symbolic and algebraic

36] M. Safey El Din and E. Schost. A nearly optimal algorithm for deciding connectivity queries in smooth and bounded real algebraic sets. arXiv preprint arXiv:1307.7836, 2013.

E. Schost. Computing parametric geometric resolutions. Applicable Algebra in Engineering, Communication and

[38] I. R. Shafarevich and K. A. Hirsch. Basic algebraic geometry, volume 197. Springer, 1977.

39] Sturm and V. Weispfenning. Computational geometry problems in REDLOG. In ADG, pages 58-96, 1996. N. Vorobjov. Complexity of computing the local dimension of a semialgebraic set. Journal of Symbolic Computation,
27(6):565 - 579, 1999. 